\title{
EL DEBATE POLITICO SOBRE LAS TERAPIAS ALTERNATIVAS Y COMPLEMENTARIAS EN ESPAÑA EN LA INTERFAZ ENTRE CIENCIA, POLITICA Y SOCIEDAD (1979-2018)
}

\author{
Dra. Emilia H. Lopera Pareja \\ Unidad de Investigación en Cultura Científica \\ Centro de Investigaciones Energéticas, Medioambientales y Tecnológicas \\ (CIEMAT), Madrid, España \\ emilia.lopera@ciemat.es \\ ORCID iD: https://orcid.org/0000-0002-2028-8932
}

Recibido el 19 de febrero de 2019

Aceptado el 6 de agosto de 2019

\section{Resumen}

El objetivo de este trabajo es investigar los procesos de construcción de los discursos políticos sobre las terapias alternativas y complementarias (TAC) en el contexto español mediante el análisis de los términos lingüísticos y los elementos narrativos utilizados en la actividad parlamentaria para referirse a este heterogéneo conjunto de tratamientos, técnicas y productos en el periodo 19792018. Para ello nos basamos en el marco teórico del coproduccionismo, desarrollado en el ámbito de los Estudios Sociales de la Ciencia por Sheila Jasanoff (2004). Mediante la aplicación de técnicas de análisis de contenido y análisis del discurso se traza la evolución de la atención política, se mapean las implicaciones léxico-semánticas de la terminología y se identifican los elementos narrativos de las TAC en la esfera política española. Según los resultados, la actividad parlamentaria se centró fundamentalmente en la acción de control al Gobierno de turno y, en mucha menor, medida en la presentación de propuestas. La mayoría de las iniciativas debatidas se plantearon desde un enfoque pluralista a la hora de articular la relación entre las TAC y la medicina convencional. No obstante, a partir de 2017 se produjo un punto de inflexión hacia posturas oposicionistas evidenciadas por el uso de los términos "pseudociencias" y "pseudoterapias" y elementos narrativos que enfatizaron la seguridad de los pacientes y la noción de víctima. Las dificultades para regular las TAC y la relevancia del conocimiento científico se evidenciaron por la continua demanda de asesoramiento experto.

Palabras clave: terapias alternativas y complementarias, TAC, pseudoterapias, pseudociencias, debate político, España. 


\title{
THE POLITICAL DEBATE ON COMPLEMENTARY AND ALTERNATIVE MEDICINE
} IN SPAIN AT THE INTERFACE OF SCIENCE, POLITICS AND SOCIETY (1979-2018)

\begin{abstract}
The aim of this paper is to investigate the construction processes of political discourses on complementary and alternative medicine (CAM) in the Spanish context through the analysis of linguistic terms and narratives used in the parliamentary activity to make reference to this heterogeneous set of treatments, techniques and products throughout the period 1979-2018. For this purpose, we build upon the theoretical framework of coproductionism, developed in the field of Social Studies of Science by Sheila Jasanoff (2004). Through the application of content analysis and discourse analysis, the evolution of political attention to the issue is traced, the lexical-semantic implications of the terminology are mapped and the narratives are identified in the Spanish political sphere. According to the results, the parliamentary activity was mainly focused on controlling the Government in power and, to a much lesser extent, on the submission of proposals. Most of the discussed initiatives addressing the relationship between CAM and conventional medicine were launched from a pluralistic approach. However, as of 2017 there was a turning point towards oppositionist positions evidenced by the use of the terms 'pseudosciences' and 'pseudo therapies', and narratives emphasizing the safety of patients and the notion of victim. The difficulties of regulating CAM and the relevance of scientific knowledge were evidenced by the continued demand for expert advice.
\end{abstract}

Keywords: complementary and alternative medicine, CAM, pseudo therapies, pseudosciences, political debate, Spain. 


\section{Introducción}

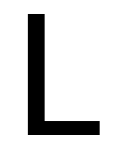

a investigación académica sobre comunicación y políticas públicas en el ámbito de la salud es un campo de trabajo amplio en el que se solapan distintas esferas sociales y sus respectivos actores, como la esfera científica, la esfera política y los medios de comunicación. A estos se suman otro tipo de agentes específicos procedentes del sector empresarial y del asociacionismo cívico y profesional, como asociaciones de pacientes o colegios o asociaciones profesionales. Las reivindicaciones y los intereses de estos colectivos tienden a visibilizarse a través de los medios de comunicación que, a su vez, actúan de correa de transmisión, no solo informativa sino también de presión política. Estas dinámicas se hacen más evidentes cuando el diseño de políticas públicas tiene por objeto temas controvertidos y polémicos sobre los que no existe un consenso generalizado porque, además del peso de la evidencia científica, también están sujetos a factores culturales como los valores y los sistemas de creencias relacionados con la salud. Este es el caso de las llamadas terapias alternativas y/o complementarias, en adelante TAC.

Las TAC consisten en una amplia y diversa gama de técnicas y tratamientos que, al igual que la medicina convencional occidental, no solo se asocian con importantes beneficios para la salud sino también con riesgos (como los efectos secundarios iatrogénicos) así como con el empeoramiento de la salud de los pacientes en el caso de algunas enfermedades graves (Han et al., 2011; Leggett, Koczwara y Miller, 2015; Patel, Kemper y Kitzmiller, 2017; Yun et al., 2013). Sin embargo, al contrario que la medicina convencional, la armonización de los aspectos teóricos y el consiguiente marco regulatorio sigue siendo un desafío relevante a escala global (OMS, 2013), en la Unión Europea (European Parliament, 2017) y, en particular, en el contexto español.

La Organización Mundial de la Salud (OMS) en su informe estratégico sobre la cuestión para el periodo 2014-2023 reconoce que en la actualidad existen importantes desafíos en este área, entre ellos, destaca: a) el desarrollo y aplicación de políticas y reglamentos, y b) la comunicación y el intercambio de información sobre estas políticas y reglamentos, así como sobre los datos de investigaciones y los recursos informativos objetivos y fiables para los usuarios (OMS, 2013). Ambos retos están estrechamente relacionados con el diseño de políticas y la comunicación política.

En países como Estados Unidos, Canadá o Chile, como caso singular por la medicina tradicional mapuche, el aumento de la demanda y uso de las TAC ha ido acompañado del desarrollo paulatino de regulaciones con objeto de garantizar la calidad, seguridad y efectividad de estas prácticas y tratamientos, así como la debida cualificación y acreditación de sus profesionales. En cambio, la situación en España se caracteriza por un vacío legal salvo un par de excepciones que, en 
cualquier caso, no incluyen la regulación de la enseñanza y acreditación para su ejercicio. La primera excepción fue la regulación mediante un Real Decreto de 2003 de las Unidades de terapias no convencionales que se definían como aquellas en que:

"Un médico es responsable de realizar tratamientos de las enfermedades por medios de medicina naturista o con medicamentos homeopáticos o mediante técnicas de estimulación periférica con agujas u otros que demuestren su eficacia y su seguridad" (Agencia Estatal Boletín Oficial del Estado, 2003: 37902).

En la actualidad existen en España 1.178 unidades de estas características, aunque el $96,5 \%$ de ellas se encuentran en centros sanitarios privados, frente al $3,5 \%$ que existen en establecimientos del sistema público de salud ${ }^{1}$. La segunda excepción tiene que ver con la legislación específica de los productos homeopáticos, regulados por Real Decreto en 1994 y, más recientemente, mediante una Orden Ministerial de 2018; en ambos casos se trató de una trasposición de carácter obligatorio de la normativa sobre homeopatía de la Unión Europea al marco legislativo nacional.

Recientemente, el 14 de noviembre de 2018, el Gobierno de España presentó el Plan para la Protección de la Salud frente a las Pseudoterapias, una iniciativa política que fija objetivos y acciones concretas dirigidos a proteger a los usuarios de las TAC. Según este documento -aún pendiente de desarrollo legislativo-, se considera pseudoterapia cualquier:

Sustancia, producto, actividad o servicio con pretendida finalidad sanitaria que no tenga soporte en el conocimiento científico ni evidencia científica que avale su eficacia y su seguridad (Gobierno de España, 2018: 3).

Epistemológicamente se considera 'medicina basada en la evidencia' (MBE) aquella que ha demostrado su eficacia, eficiencia y seguridad mediante ensayos clínicos aleatorizados, aunque el propio concepto de MBE, nacido en los años noventa del siglo pasado, está sujeto a continua revisión (Kohatsu, Robinson y Torner, 2004).

Desarrollado conjuntamente por el Ministerio de Sanidad, Consumo y Bienestar Social y el Ministerio de Ciencia, Innovación y Universidades, este plan del Gobierno de España es, en cualquier caso, una iniciativa política relevante por múltiples razones, entre las que destacan las siguientes: en primer lugar, concierne al ámbito de la salud pública, por lo que afecta al conjunto de la sociedad y a los profesionales de las TAC y de la medicina convencional; en

\footnotetext{
${ }^{1}$ Datos en julio de 2018 según el Registro General de centros, servicios y establecimientos sanitarios del Ministerio de Sanidad del Gobierno de España. Disponible en:

http://regcess.mscbs.es/regcessWeb/inicioBuscarCentrosAction.do
} 
segundo lugar, no existe un marco regulatorio europeo global que marque la pauta a seguir por los Estados miembros en el seno de la Unión Europea, salvo en el caso de la homeopatía que, como ya se ha dicho, cuenta con una legislación propia; tercero, el diseño de políticas públicas sobre TAC en España es una cuestión que viene de lejos pero que ningún gobierno abordó globalmente durante las últimas cuatro décadas.

Pero una de las dificultades para legislar las TAC, incluso para investigar el fenómeno desde las ciencias sociales, reside precisamente en lo que Schreiber denomina "el problema con la terminología terapéutica" (2005: 175), en clara referencia a la importancia que tiene en este tema -y en muchos otros- la precisión en el lenguaje a la hora de seleccionar determinados significantes, ya sea en un debate político para legislar o en una noticia para informar. La propuesta o elección de un término u otro por parte de los participantes en el debate define, en parte, el discurso sobre la materia al tiempo que marca los límites del propio debate, condicionando así el posible resultado del mismo; sea la adopción de unas políticas con determinada orientación o, en ausencia de consenso, el mantenimiento del status quo previo al debate.

Asumiendo, por tanto, que la elección de determinadas palabras para denominar determinados fenómenos modela el discurso como ejercicio de poder simbólico, el objetivo general de este trabajo es analizar cómo se ha ido modelando el discurso sobre las TAC en el contexto español a partir de la terminología y de los elementos narrativos utilizados para hacer referencia a la materia en sede parlamentaria en el periodo 1979-2018. En concreto se persiguen los siguientes objetivos específicos: 1) conocer la evolución, tipología y contenido de la atención política dedicada a la materia; 2 ) identificar los términos (significantes) que han ido dando sentido al discurso de las TAC en el ámbito político; y, 3) extraer los elementos narrativos que han marcado la actividad política sobre esta cuestión.

Para dar respuesta a estas cuestiones se toma como marco general la teoría del coproduccionismo desarrollada en el ámbito de los Estudios Sociales de la Ciencia (Jasanoff, 2004). Se trata de una aproximación conceptual integrativa e interdisciplinar adecuada para el análisis social de los procesos que combinan construcción de conocimiento y ordenamiento social mediante leyes, grupos de expertos, grupos de interés y representaciones mediáticas. Como instrumento se recurre al análisis del discurso porque precisamente los discursos y sus contenidos, tácitos y no tácitos, son uno de los instrumentos de coproducción simétrica de la construcción del conocimiento y el ordenamiento social.

\section{Antecedentes}

El creciente interés por las TAC desde las ciencias sociales se sitúa en el cambio de siglo, coincidiendo con la atención dedicada desde los círculos clínicos y desde las políticas en salud pública (Brosnan, Vuolanto y Dannell, 2018). A través de la lente de diferentes disciplinas y marcos conceptuales se ha ido desarrollado 
un corpus de literatura en el que se abordan las diversas dimensiones sociales que se han visto afectadas por la convivencia de las TAC con la medicina convencional en los sistemas de atención sanitaria. Entre las cuestiones que han recibido atención desde el ámbito académico destacan, entre otras, las siguientes: 1) los estudios sobre el aumento de la demanda y uso de las TAC, así como los motivos subyacentes; 2) las distintas formas de articulación entre las TAC y la medicina convencional; 3) el papel de los medios de comunicación, de internet y de las redes sociales; $y, 4)$ la dimensión política y su relación con el conocimiento científico.

En cuanto al uso, los datos apuntan a un rápido crecimiento en los países occidentales, especialmente en Estados Unidos (Honda y Jacobson, 2005) y Europa, donde 100 millones de europeos utilizan este tipo de tratamientos (OMS, 2013). Diferentes encuestas han puesto de manifiesto que su uso también se ha extendido en España durante la última década. Así lo indica la Encuesta Nacional de Salud del Ministerio de Sanidad español, según la cual un 4,8\% de la población ha visitado a un profesional de las TAC en el último año (Ministerio de Sanidad, 2013). Otra encuesta más reciente del Centro de Investigaciones Sociológicas ha desvelado que entre el $20,4 \%$ y el $9,8 \%$ de la población española reconoce haberse tratado con alguna de las cuatro TAC más utilizadas en España -masaje terapéutico, remedios herbales, meditación y homeopatía (CIS, 2018).

Entre las motivaciones para su consumo destacan la prevalencia de trastornos y enfermedades que no son fácilmente tratables por la medicina convencional, como los estados psicológicos de ansiedad y la depresión, o las enfermedades crónicas como el SIDA (Cauffield, 2000; Webb et al., 2018). Los datos han demostrado que el uso de TAC no se debe fundamentalmente a la insatisfacción con la medicina convencional (Eisenberg et al., 2001), aunque los profesionales de las TAC consideran que ellos ofrecen una atención personalizada que, por lo general, los pacientes no suelen recibir por parte de los profesionales de la medicina convencional (Geist-Martin y Bell, 2009).

En cuanto a los modelos para articular la convivencia entre las TAC y la medicina convencional, Kaptchuk y Miller (2005) distinguen tres modelos éticos: oposicionista, integrativo y pluralista. Según el modelo oposicionista, el ejercicio de la medicina debe eliminar las TAC por el bien de los pacientes; el enfoque integrativo defiende un uso combinado de la medicina convencional y de las TAC con el objetivo de promover el bienestar y tratar las enfermedades teniendo en cuenta tanto sus manifestaciones físicas como las emocionales y espirituales; por último, estos autores se decantan por un modelo pluralista que, en sus propias palabras, significa "que la medicina convencional y las TAC deben relacionarse entre sí como sistemas médicos independientes pero cooperativos" (2005: 286).

El modelo pluralista está en sintonía con la actual estrategia de la OMS sobre medicina tradicional para el periodo 2014-2023 (OMS, 2013). A través de este 
documento, la OMS manifiesta que la medicina tradicional y complementaria, integrada en las TAC, constituye una parte importante y a menudo subestimada de la atención sanitaria. En consecuencia, la actual estrategia del máximo organismo internacional de la salud tiene como objetivo apoyar a todos los Estados miembros en el desarrollo de políticas públicas proactivas dirigidas a fortalecer el papel de estas terapias para contribuir a la salud de la población.

Por su parte, entre los resultados de la investigación en comunicación sobre TAC se incluyen los siguientes hallazgos. Un metaanálisis realizado por Weeks y Strudsholm (2008), a partir de los resultados de una muestra de dieciséis trabajos con datos de diferentes países, concluyó que la atención mediática había aumentado en la primera década del siglo XXI -excepto en Norteamérica-, era más positiva que negativa aunque no exenta de controversia e incluía el debate sobre la evidencia científica. En el contexto español no se han encontrado evidencias de la relación entre el volumen de cobertura informativa y la frecuencia de uso de estas terapias (Moreno y Lopera, 2016). Como el uso tampoco parece estar relacionado con el volumen de consultas en internet (Cano-Orón, 2016), se concluyó que la decisión de usar estas terapias puede deberse más al boca a boca que al impacto mediático. Otros trabajos han revelado que la presencia de la evidencia científica como argumento periodístico está infrarrepresentada en los medios españoles, aunque la mayoría de las noticias presenta un enfoque negativo (Moreno Tarín, 2019), especialmente en el caso de la homeopatía (Escriba-Sales, Rovira y Alonso-Marco, 2015). Por último, Moreno Tarín (2019) señaló que, entre 2015 y 2017, el significante 'pseudociencias' fue el más utilizado en la esfera mediática para referirse a estas terapias, seguido a mucha distancia de 'terapias alternativas', 'medicina alternativa' y 'pseudoterapias'.

En cuanto a la dimensión política, la investigación se ha centrado en esclarecer cómo el ejercicio de las TAC es regulado mediante el diseño de políticas públicas que varían en función del contexto nacional. Se trata de un territorio tensionado donde los representantes políticos deben intermediar entre las demandas de los pacientes, las de los profesionales de las TAC y de la medicina convencional superando, al mismo tiempo, las presiones de los grupos de interés tanto del sector sanitario como de las organizaciones escépticas (Brosnan y Kirby, 2016). Entre los hallazgos de esta línea de investigación destaca el fenómeno de la glocalización (Almeida, Siegel y Barros, 2018), que consiste en que las distintas modalidades de TAC se extienden a nivel global, en cuanto al conocimiento que se tiene de ellas, pero las condiciones para su ejercicio varían mucho en función de los contextos nacionales en los que se establecen políticas públicas para su regulación. En España, se analizó el posicionamiento de los diferentes partidos políticos ante las TAC en la campaña electoral de diciembre de 2015 según el tratamiento que dieron a esta materia en sus programas electorales y en Twitter (Cano-Orón y Moreno, 2017). Según las autoras, este tema no estuvo presente en Twitter y se mencionó muy someramente en los programas electorales. Mientras que el Partido Popular y Podemos no mencionaron las TAC y, por tanto, no se 
posicionaron al respecto, Unidad Popular se refirió a ellas como "procedimientos terapéuticos pseudocientíficos" y Ciudadanos, como "estrategias o prácticas clínicas desaconsejadas por la evidencia científica". Ambos partidos se mostraron partidarios de eliminarlas del Sistema Nacional de Salud. Por el contrario, el PSOE las denominó "terapias naturales" y proponía regularlas para garantizar su calidad a las personas que eligieran utilizarlas (Cano-Orón y Moreno, 2017: 176).

Como ámbito clave de la gobernanza política, los debates en sedes parlamentarias también se han analizado en tanto que actividad donde se producen discursos orales y escritos que tienen como objetivos legitimar u oponerse a ciertas demandas mediante la legislación, representar los diversos intereses y controlar la actividad del Gobierno e influir en las opiniones (Bayley, 2004). Este fue el escenario elegido por Danell (2018) para analizar el debate político sobre las TAC en el Parlamento sueco en el periodo 1980-2015 con objeto de comprobar cómo se formuló esta materia como problema político que requería soluciones.

\section{Aproximación teórica}

Esta investigación se encuadra dentro del campo de los Estudios Sociales de la Ciencia (Estudios CTS) que investiga la relación entre la ciencia y la sociedad. Los Estudios CTS han realizado valiosas aportaciones tanto desde el análisis de las políticas públicas para el desarrollo de la ciencia y la tecnología (policy for science), como desde el estudio del papel de la ciencia y la tecnología en la regulación de cuestiones sensibles relacionadas, por ejemplo, con la salud pública o el medio ambiente (science for policy) (Brooks, 1964). Dentro de esta segunda categoría, los Estudios CTS han centrado su atención en temas cuyo abordaje desde las políticas públicas resultaba complejo y/o controvertido. Tal complejidad y/o controversia a la hora de legislar puede obedecer a múltiples causas como, por ejemplo, al alto componente de información científico-técnica que implica una materia, la falta de consenso social y/o político -entre partidos y en el seno de los propios partidos-, los valores en disputa y sus implicaciones éticas o la percepción del riesgo. El interés académico que suscitan estas cuestiones lo resume muy bien Abel Novoa en su reseña sobre el libro Campañas institucionales en salud pública. El caso de la vacuna contra el virus VPH, editado por Moreno (2016):

"Desde el punto de vista sociológico, las controversias son sitios privilegiados de observación porque revelan la fabricación política y social de la ciencia" (Novoa, 2019: web).

Esta idea general subyace en el capítulo introductorio del libro State of knowledge. The coproduction of science and social order, editado por la filósofa de la ciencia Sheila Jasanoff (2004), en el que esta autora hace un repaso del corpus de investigación que ha demostrado que la ciencia y la tecnología se 
pueden estudiar como prácticas sociales orientadas al establecimiento de varios tipos de estructura y autoridad. En este sentido, la autora explica que:

"[...] las realidades que experimentamos los seres humanos son fruto de una empresa científica, técnica y social: es decir, ciencia y sociedad son coproducidas, respaldándose la una en la otra" (Jasanoff, 2004: 17).

Ahora bien, esto no significa que la ciencia sea construida socialmente, unidireccionalmente, sino que el fenómeno "relación ciencia-sociedad" se desarrolla simétricamente. Estos son los términos en que Jasanoff plantea la teoría coproduccionista como una aproximación conceptual integrativa e interdisciplinar adecuada para el análisis de cuestiones, objetos de investigación o temas que implican procesos sociales que combinan la construcción del conocimiento y el ordenamiento social mediante el desarrollo de leyes, la participación de grupos de expertos, de grupos de interés y las representaciones mediáticas. En el caso de las TAC, el devenir de estos procesos sociales podría conducir al establecimiento de una regulación (o al mantenimiento del status quo de materia no regulada) sobre, al menos, los siguientes aspectos: en primer lugar, su consideración en la atención sanitaria (como una oportunidad o como un peligro); en función de ello, si procede, se regularían otras cuestiones derivadas como, por ejemplo, los requisitos de formación y las condiciones para el ejercicio profesional.

El presente trabajo de investigación se puede enmarcar dentro de una rica producción académica que sirve de referencia a la hora de analizar los procesos comunicativos relacionados con el debate social sobre la salud y la enfermedad. Gran parte de la literatura resultante de estas investigaciones procede de autores y grupos de investigación con una dilatada trayectoria centrada en el estudio de la representación social de temas concretos relacionados con la salud, como las enfermedades raras, la discapacidad, las vacunas o las TAC. Entre ellos destacan el Grupo ECCO (Estudios Críticos sobre la Comunicación), de la Universidad de Almería, el grupo GIDYC (Grupo de Investigación sobre Discapacidad y Comunicación), de la Universidad CEU-Cardenal Herrera de Valencia, y el Grupo ScienceFlows, de la Universitat de València. Algunas de sus contribuciones más relevantes se recogen en las siguientes publicaciones colectivas: 1) un monográfico realizado por los grupos de investigación ECCO y GIDYC, titulado Desafíos y estrategias comunicativas de las enfermedades raras: la investigación médica como referente (Bañón et al., 2011); 2) el número especial "Salud y discurso" de la revista Discurso \& Sociedad (VV.AA., 2013); y, 3) un monográfico realizado por el grupo ScienceFlows, titulado Campañas institucionales en salud pública. El caso de la vacuna contra el virus VPH (Moreno, 2016).

Desde el marco conceptual del coproduccionismo se asume que existe una "interacción constante entre lo cognitivo, lo material, lo social y lo normativo" (Jasanoff, 2004: 38), o lo que es lo mismo, entre la capacidad humana de producir 
conocimientos y la capacidad humana para producir aparatos que ordenan y reordenan la sociedad. Tal interacción tiene como denominador común necesario los flujos comunicativos que se producen, entre las distintas esferas sociales científica, política, mediática, etc.-, articulados mediante discursos. Precisamente la creación de discursos es identificada por Jasanoff como uno de los cuatro instrumentos más frecuentes de los procesos de coproducción, junto con la creación de identidades, instituciones y representaciones. Esta es la razón por la que en este trabajo analizamos los discursos de las TAC en la esfera política, concretamente en las iniciativas parlamentarias registradas por los grupos políticos en las Cortes Generales del Estado español.

El interés por analizar las TAC en el entorno político se fundamenta en el modelo de las esferas públicas de Hilgartner y Bosk (1988). Según este trabajo seminal, la esfera política sería uno de los ámbitos sociales donde los diversos temas o problemas aparecen y desaparecen debido a dinámicas de competición y de selección entre los mismos. Estas dinámicas serían similares a las que rigen la competencia entre especies en los nichos ecológicos de los ecosistemas naturales. Según estos autores, el proceso de selección se produce simultáneamente a dos niveles. Como las esferas públicas tienen una capacidad de carga finita, en el primer nivel múltiples asuntos y problemas compiten por introducirse y mantenerse en la esfera, acaparando espacio, atención y recursos que varían según el tipo de esfera; desde ser objeto de proyectos de investigación (esfera científica), de tratamiento periodístico (esfera mediática) o de debate político (esfera política). En el segundo nivel, la competición se produce entre las distintas formas de presentar el asunto o problema. $Y$ es en este segundo nivel donde el discurso modula la interacción entre lo cognitivo, lo material, lo social y lo normativo, reiterando las palabras de Jasanoff.

Según el lenguaje coproduccionista, el análisis de los discursos sería uno de los recursos disponibles para investigar los procesos de creación de significado en un mundo en el que la ciencia y la tecnología son cada vez más omnipresentes tanto para el ciudadano, en su vida diaria, como para los políticos en su labor legisladora. Al tratarse "de un marco de trabajo integrativo e interdisciplinar" (Jasanoff, 2004: 43), la coproducción permite -y se nutre de- aportaciones desde ámbitos diferentes como la antropología y la historia, la legislación y las políticas públicas, los estudios culturales y la teoría social. Sirviéndonos de la versatilidad que ofrece la aproximación teórica coproduccionista como marco general, este estudio recurre al análisis del discurso entendiendo este como marco teórico además de herramienta metodológica- específico.

Desde el punto de teórico, el análisis del discurso se enmarca dentro de los estudios de la semiótica social que se ocupan de la utilización de los recursos semióticos en las prácticas sociales. En su sentido más amplio, son recursos semióticos tanto las acciones como los artefactos que usamos para comunicarnos, bien de forma fisiológica -con las cuerdas vocales o por gestos- o bien mediante 
tecnologías -un bolígrafo, un papel o un ordenador- (Leeuwen, 2005). Como herramienta metodológica, el análisis del discurso se puede clasificar dentro del análisis de contenido cualitativo pero, al contrario que este, va un paso más allá del análisis formal de los textos para intentar encontrar relaciones entre los textos y los contextos en los que tiene lugar su producción.

Como aproximación teórica, el discurso se contempla como un tipo de práctica social que influye en el contexto social y, al mismo tiempo, es permeable al mismo. Esta retroalimentación da lugar a nuevas formas de pensar en relación a una cuestión, un objeto de investigación o tema, conformando así las interpretaciones que hacemos de los mismos y, lo más importante, nuestra forma de actuar sobre ellos. Todos estos procesos sociales complejos y bidireccionales que modelan el discurso en un contexto determinado no suelen obedecer al azar, más bien al contrario. En este sentido, la literatura ha destacado que el interés del análisis del discurso estriba precisamente en evidenciar que, por lo general, los intereses en juego en cada discurso obedecen a ideologías. Como sostiene Dijk:

Además de su función social de sostener los intereses de los grupos, las ideologías tienen la función cognitiva de organizar las representaciones (actitudes, conocimientos) sociales del grupo, y así monitorizar indirectamente las prácticas sociales grupales, y por lo tanto también el texto y el habla de sus miembros (2008: 208).

Es decir, a través de los discursos las ideologías intervienen en la creación de significados $\mathrm{y}$, por ende, en la construcción del conocimiento y en el ordenamiento social. Relacionado directamente con las ideologías y el control social, Leeuwen (2005) también ha destacado que, en la vida en sociedad, las personas -por extensión, partidos políticos, grupos de interés, etc.- intentan constantemente fijar y controlar el uso de los recursos semióticos que constituyen el discurso. Gran parte del diseño y control del discurso se lleva a cabo mediante la selección de recursos semióticos cuya interpretación propicia una determinada forma de actuar de acuerdo con los intereses de determinados grupos. Aunque este autor reconoce que esta situación se da más en unos ámbitos que en otros, consideramos que los contextos en los que tiene lugar la negociación política que implica el debate y la aprobación de leyes y normas orientadas al ordenamiento social, son un terreno muy sensible y fértil para analizar estas dinámicas.

Más centrado en el análisis de los textos producidos por los medios de comunicación como arena pública en la que se negocian los significados a la hora de alcanzar un consenso social sobre lo que es o no un problema, Entman (2004) entiende el discurso como un marco de referencia que se construye mediante la selección de palabras clave, metáforas y conceptos, con objeto de alentar a los receptores a interpretar determinados acontecimientos y temas de determinada manera. 
Pérez (2008) plantea el análisis del discurso como marco teórico y metodológico para aplicar en el campo de la investigación en comunicación basándose en las aportaciones a la semiótica de Roland Barthes y Ferdinand de Saussure. Según esta autora, desde el enfoque barthesiano, el discurso es un fenómeno del campo de la comunicación puesto que captura la imaginería creada y compartida en torno a una cuestión, un objeto de investigación o un tema, entendiendo como 'imaginería' "el acervo común de sentidos socialmente creados y compartidos" (Pérez, 2008: 227) sobre estos. En consecuencia, el estudio de la imaginería en torno a un determinado objeto, en determinados textos y contextos, ayudaría a entender cómo se concibe el objeto y, lo que resulta aún más relevante, qué acciones cabría esperar en función de cada concepción del mismo. Trasladado esto al caso de estudio que nos ocupa, el análisis de los discursos de las TAC en el debate parlamentario revelará la imaginería utilizada por los representantes políticos para referirse a este tema, al tiempo que nos ayudaría a comprender cómo determinadas formas de concebir las TAC -es decir, de denominarlasimplican determinados cursos de acción en su regulación.

Transitando desde el plano teórico al metodológico, el acervo común de sentidos socialmente creados y compartidos sería, pues, un "repertorio de imágenes constituidas como 'unidades de sentido' que dan, precisamente, sentido al decir y al hacer común sobre un objeto" (Pérez, 2008: 231). Basándose en la semiótica de Saussure, esta autora explica que las unidades de sentido, como recurso semiótico, se plasman en signos que, a su vez, están conformados por un significante y un significado. Así, por ejemplo, en un texto lingüístico, los significantes serían el conjunto de las palabras para referirnos a un objeto; pero cada una de esas palabras tendría asociado un significado, que sería el sentido o la connotación elegida en cada caso para referirse a tal objeto.

Sobre la importancia de la precisión en el lenguaje en la investigación en comunicación referida a las TAC, Schreiber (2005) identifica de manera exhaustiva la terminología que se usa en Estados Unidos. Concluye que existen importantes distinciones entre el uso de términos como 'holístico' y 'alternativo' en función de cómo se construyen y evalúan los diferentes sistemas médicos. Según esta autora, el uso de términos como 'biomédico' u 'holístico' se refiere a modelos explicativos diferentes que, como tales, tienen consecuencias en el discurso. Gale (2014) sostiene que el propio proceso de denominación de las TAC da una idea de la complejidad que caracteriza la materia desde el poder y la historia. Como explica Díaz Rojo (2006) en su artículo Cómo denominar a las "otras" medicinas:

La variada terminología es reflejo de la diversidad y confrontación de los sectores médicos en pugna, además de ser producto de la desigual naturaleza y estatus de los diferentes sistemas y terapias complementarias. La complejidad de estas medicinas produce rechazos y prejuicios mutuos, posturas de prepotencia, abusos y fraudes, lo que hace que reine la confusión en la sociedad en torno a la eficacia y 
fiabilidad de las terapias situadas al margen de la medicina científica (Díaz, 2006: web).

Aplicado a este estudio de caso, los diferentes "cursos de acción" serían los tres modelos éticos que, según Kaptchuk y Miller (2005), caracterizan la relación entre las TAC y la medicina convencional -oposicionista, integrativo y pluralista- y cuyas características se han explicado anteriormente en el apartado de este artículo dedicado a los antecedentes.

En resumen, desde esta aproximación teórica híbrida entre el coproduccionismo de los Estudios CTS y el análisis del discurso de la semiótica, planteamos analizar el debate de las TAC en España tomando como objeto de estudio los textos registrados sobre esta cuestión en sede parlamentaria en el periodo 1979-2018. El objetivo último es evidenciar cómo los distintos discursos de las TAC en el ámbito político a lo largo de los últimos cuarenta años han "coproducido" la relación entre dos visiones de la atención sanitaria -la medicina convencional y las TAC- mediante la utilización de diferentes "unidades de sentido", constituidas por un significante y un significado, y elementos narrativos. Así, pues, atendiendo a la terminología y a los relatos usados para referirse a las TAC en la comunicación política desde una perspectiva diacrónica se podrá determinar qué modelos éticos han prevalecido y, lo más importante, qué consecuencias normativas se han derivado de los mismos.

\section{Método}

El marco temporal fijado abarca desde marzo de 1979 a diciembre de 2018, es decir, las doce últimas legislaturas de la democracia española sin contar el periodo dedicado exclusivamente a la redacción y aprobación de la actual Constitución de 1978. El corpus de análisis lo constituyen los expedientes parlamentarios sobre las TAC registrados durante este periodo en las Cortes Generales.

Para acceder a esta documentación como fuente de datos, se procedió a la identificación y recopilación de los expedientes parlamentarios utilizando los buscadores en línea de las dos cámaras de las Cortes Generales -Congreso de los Diputados $^{2}$ y Senado ${ }^{3}$ - que permiten realizar búsquedas sistemáticas mediante palabras clave. Como palabras clave se utilizaron los términos 'terapias naturales', 'terapias alternativas', 'terapias complementarias', 'medicinas no convencionales' y 'terapias no convencionales', similares a los utilizados por Danell (2018) para sus búsquedas en el Parlamento sueco sobre esta cuestión. También se incluyeron los términos 'pseudoterapias' y 'pseudociencias' porque en los últimos años en

\footnotetext{
2 Buscador del Congreso de los Diputados:

http://www.congreso.es/portal/page/portal/Congreso/Congreso/Iniciativas

3 Buscador del Senado:

http://www.senado.es/web/actividadparlamentaria/iniciativas/buscadoriniciativas/index.html
} 
España ha sido una denominación muy común en la prensa digital para referirse a las TAC (Moreno, 2019). Por último, se añadieron 'homeopatía' y 'acupuntura' porque son las terapias que, junto con el yoga, tienen una mayor presencia en la prensa digital española (Moreno y Lopera, 2016).

Mediante este procedimiento de búsqueda se obtuvo un total de 80 expedientes: 60 en el Congreso de los Diputados y 20 en el Senado. Cada expediente parlamentario se tomó como una unidad de análisis. Por tanto, el corpus de análisis quedó compuesto por 80 unidades que se distribuyen en distintas categorías de iniciativas parlamentarias. En concreto, entre seis de las recogidas en los reglamentos de ambas cámaras legislativas: a) preguntas escritas al Gobierno; b) preguntas orales en Pleno; c) proposiciones no de ley; d) proposiciones de ley; e) comparecencias en comisión; y, f) mociones. A continuación se explica en qué consiste cada modalidad.

Las preguntas escritas al Gobierno y, su contestación también por escrito, se publican en el Boletín Oficial de las Cortes Generales del Congreso de los Diputados y del Senado, según cada caso. Las preguntas orales en Pleno del Congreso, en cambio, son contestadas verbalmente por el titular del ministerio correspondiente y su transcripción se publica en el Diario de Sesiones del Congreso de los Diputados. En ambos casos, se trata de iniciativas dirigidas al control de las actuaciones del Ejecutivo.

Asimismo, las proposiciones no de ley (PNL) consisten en propuestas presentadas por los grupos parlamentarios que se someten a proceso de enmiendas y a debate antes de proceder a su votación. Aunque salgan aprobadas, al no tener carácter de ley, no son vinculantes. Se trata de textos sobre una materia concreta sobre la que: a) se insta al Gobierno a que realice una actuación; b) se definen las bases o principios de actuación de las Administraciones Públicas; o, c) se da la opinión mayoritaria de las Cortes Generales al respecto. Por su parte, las proposiciones de ley (PDL), al contrario que las $\mathrm{PNL}$, son iniciativas con pleno carácter legislativo y de carácter vinculante una vez que son aprobadas mediante votación. Mientras que los proyectos de ley los presenta el Gobierno, las PDL las puede presentar un único diputado con la firma de otros catorce, o un grupo parlamentario con la firma de su portavoz.

Por otra parte, las comparecencias pueden realizarse en el Pleno o en comisión. Por regla general, son los titulares de los departamentos ministeriales los que suelen comparecer en Pleno, bien a petición propia o por solicitud de la oposición, mientras que a las comisiones se suelen convocar a otras autoridades, funcionarios o personalidades especialmente competentes en una materia específica para que informen y asesoren a los legisladores de dicha comisión. Por último, las mociones presentadas en el Senado pueden tener distintas finalidades, desde instar al Gobierno a que formule una declaración o remita al Congreso un 
proyecto de ley regulando una materia, hasta que la Cámara delibere y se pronuncie sobre un texto de carácter no legislativo.

Si atendemos a las características de los textos analizados descritas en los párrafos anteriores, el diseño de esta investigación sobre el debate político de las TAC en el contexto español se enmarcaría en un nivel de análisis denominado macrodiscursivo, es decir, aquel que "se ocupa de las interacciones de grandes grupos, tales como las que se desarrollan en el Parlamento cuando se gestiona alguna norma o ley relacionada con la salud" (Bañón, 2013: 15).

Una vez construido el corpus, el diseño de la investigación se estructuró en dos partes, una cuantitativa y otra cualitativa (ver Tabla 1). En primer lugar se cuantificó el número de expedientes parlamentarios registrados cada año como indicador de la atención política dedicada a las TAC. La atención política se puede definir como el interés, la preocupación y e incluso el nivel de urgencia de los representantes políticos por este tema; es decir, el nivel de relevancia y prioridad que se le otorga (Hilgartner y Bosk, 1988). La monitorización del comportamiento de este indicador permite apreciar cómo se distribuye la atención política desde una perspectiva diacrónica y, por tanto, resulta útil para determinar la ausencia o presencia de las TAC en la esfera política en el periodo analizado y el nivel de actividad alcanzado.

Por lo que respecta a la parte cualitativa del diseño de la investigación, el análisis del discurso aplicado al corpus se estructuró en dos apartados diferenciados, a la par que complementarios: a) un análisis léxico-semántico de la terminología utilizada; 2) un análisis de los elementos narrativos más destacados. A continuación se describe en qué consiste cada parte.

El primer tipo de análisis se abordó considerando cada unidad de análisis, es decir, cada expediente originado en sede parlamentaria sobre este tema. El análisis léxico-semántico alude, por tanto, al registro y monitorización de la elección de distintas palabras o expresiones (significantes) para referirse a las TAC (con un determinado significado) y, por lo tanto, asumiendo que la elección de cada significante implica unas connotaciones de sentido e intencionalidad en los textos, tal y como se ha explicado en el epígrafe dedicado al marco conceptual de la investigación. 
Tabla 1. Resumen del diseño metodológico.

\begin{tabular}{|c|c|c|}
\hline \multirow{8}{*}{$\begin{array}{l}\text { Análisis } \\
\text { cuantitativo }\end{array}$} & \multicolumn{2}{|c|}{ Datos de la unidad de análisis } \\
\hline & Fecha de la iniciativa & --/--/---- \\
\hline & $\mathrm{N}^{\circ}$ de expediente & --/-------- \\
\hline & Legislatura (I-XII) & $|-X| I$ \\
\hline & Cámara & Congreso/Senado \\
\hline & Tipo de iniciativa & Pregunta/PNL/comparecencia/... \\
\hline & Partido proponente & PP/PSOE/CiU/Ciudadanos/... \\
\hline & Promotor de la iniciativa & Gobierno/oposición \\
\hline \multirow{5}{*}{$\begin{array}{l}\text { Análisis } \\
\text { cualitativo }\end{array}$} & \multicolumn{2}{|c|}{ Análisis léxico-semántico (nivel microestructural) } \\
\hline & $\begin{array}{l}\text { Significantes específicos } \\
\text { TAC }\end{array}$ & Homeopatía/acupuntura/... \\
\hline & Significantes generales TAC & $\begin{array}{ll}\text { [Terapias] } & \text { Naturales/alternativas/no } \\
\text { convencionales/... } & \\
\end{array}$ \\
\hline & \multicolumn{2}{|c|}{ Análisis de elementos narrativos (nivel macroestructural) } \\
\hline & \multicolumn{2}{|c|}{ Identificación de los principales argumentos, aspectos e ideas } \\
\hline
\end{tabular}

Fuente: elaboración propia.

Con objeto de detectar estrategias de generalización en el discurso de las TAC, en la codificación se distinguió entre dos tipos de significantes: significantes específicos (SE) y significantes generales (SG). En el primer caso se menciona una o más TAC de manera concreta como, por ejemplo, acupuntura u homeopatía, etc. En el segundo caso, se recurre a la utilización de una expresión como, por ejemplo, 'terapias naturales', para referirse de manera general a todas las TAC, es decir, sin establecer distinciones entre ellas. La generalización consiste en abordar las TAC como un conjunto homogéneo de terapias, tratamientos, técnicas y productos, lo que discursivamente deja escaso margen para las especificidades y los matices, tan necesarios en un debate político. Por tanto, el SG seleccionado en cada caso por los participantes en el discurso evidencia una intencionalidad en relación a la materia a regular.

Con el segundo tipo de exploración, la de los elementos narrativos, se buscaron las ideas y aspectos que emergen como argumentos narrativos dominantes en la actividad parlamentaria sobre las TAC. Según el origen del corpus, cuando los textos analizados proceden de medios de comunicación se suele hablar de análisis de los elementos narrativos o relatos (narratives) (McComas y Shanahan, 1999), y cuando estos proceden del ámbito parlamentario también se les ha denominado "regularidades discursivas" (discoursive regularities) (Mulkay, 1993). Como ha demostrado la literatura sobre las TAC (ljaz et al., 2015), este tipo de exploración permite una mejor comprensión de los términos en que se desarrolla y construye el debate político al tiempo que identifica qué partidos políticos contribuyen al debate. Se trata, por tanto, de una fórmula para mapear las narrativas hegemónicas de las TAC y sus promotores en la esfera política. 


\section{Resultados}

\subsection{Atención política: evolución, tipología y contenido}

Durante los 39 años del periodo analizado se tramitaron 80 expedientes parlamentarios sobre las TAC en las Cortes Generales del Estado español con una media de dos iniciativas por año, distribuidos como se puede ver en la Figura 1. Teniendo en cuenta que la primera iniciativa no se registró hasta 1984, se pueden distinguir tres etapas en el ciclo de atención política dedicada a la materia, cuantificando como tal el número de expedientes parlamentarios registrados por año: a) de 1984 a 2006; b) de 2007 a 2010; c) de 2011 a 2018. Durante la primera fase, las TAC aparecieron en el debate político de manera muy intermitente y con un número muy reducido de iniciativas. Sin embargo, la situación cambió radicalmente en la segunda fase, cuando se produjo un aumento sin precedentes de la atención parlamentaria. Por último, en la tercera etapa el tema recupera su intermitencia anual aunque en 2017 y 2018 se registraron valores superiores a la media.

Fuente: elaboración propia a partir de los archivos de las Cortes Generales del Estado español.

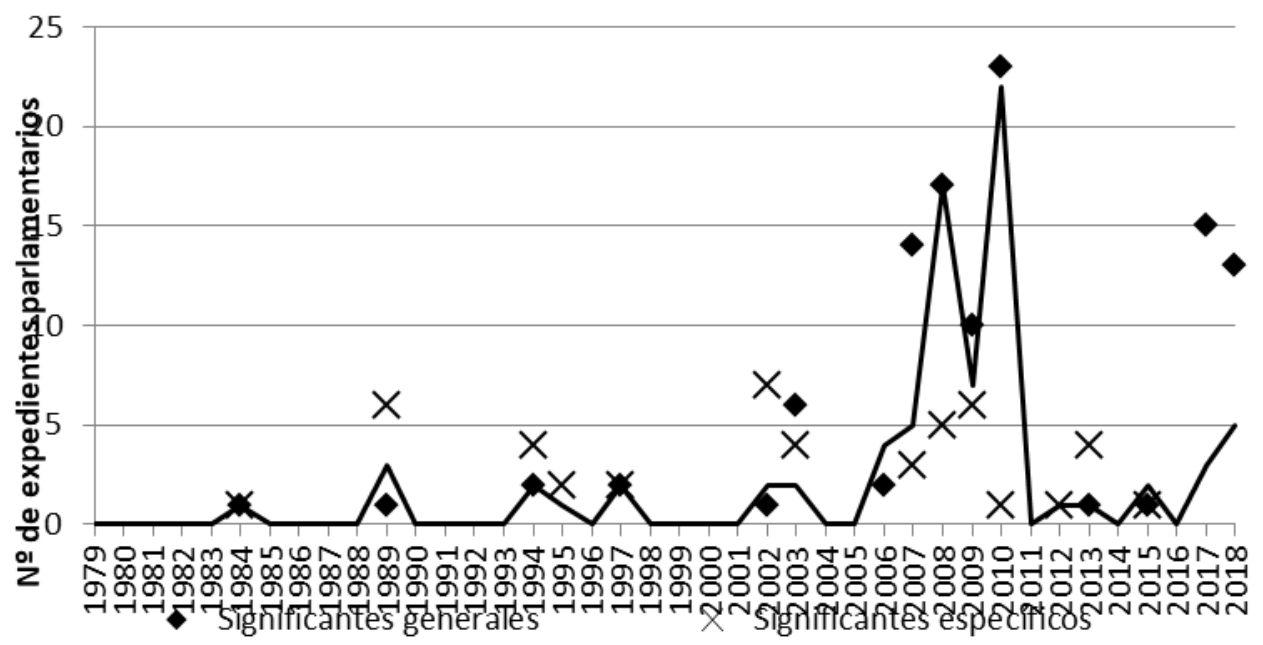

Figura 1. Evolución del volumen de expedientes parlamentarios sobre las TAC en la Cortes Generales del Estado español (1979-2018).

Por tipos de expediente (ver Figura 2), la inmensa mayoría de ellos fueron preguntas escritas de control al Gobierno de turno (79\%), seguidas a mucha distancia de las proposiciones no de ley (PNL) (10\%), las comparecencias de expertos y ministros relacionados con el tema (5\%) y las preguntas orales (4\%). También se registró una proposición de ley (PDL) y una moción. Por tanto, cuantitativamente, los hallazgos revelan que el debate político sobre las TAC se materializó fundamentalmente mediante la acción de control al Gobierno, con preguntas escritas; $y$, en mucha menor medida, mediante PNL O PDL, las 
iniciativas parlamentarias más orientas al enriquecimiento del debate puesto que son las que permiten la formulación de nuevas propuestas sobre la materia.

Las PNL y PDL que se plantearon fueron las siguientes. En primer lugar, en 1989 el PSOE -de corte socialdemócrata- solicitó al Gobierno de su mismo partido que regulara la acupuntura y la homeopatía. Estando también el PSOE en el Gobierno, pero 20 años después, este mismo grupo solicitó nuevamente que se legislaran las condiciones para el ejercicio de la homeopatía, pero especificando que los profesionales sanitarios debían ser licenciados en medicina. En 2006, el grupo de izquierdas IU-ICV fue un paso más allá y pidió la regulación e integración de las TAC en el sistema sanitario. Al año siguiente, este mismo grupo político consiguió que se aprobara una PNL para iniciar el estudio y la elaboración de una propuesta de regulación del sector. Esa tarea la desarrolló durante varios años un grupo de expertos creado ad hoc y los resultados quedaron recogidos en el informe Análisis de la situación de las terapias naturales (Ministerio de Sanidad, 2011), cuyos resultados nunca llegaron a concretarse en una regulación.

Fuente: elaboración propia a partir de los archivos de las Cortes Generales del Estado español.

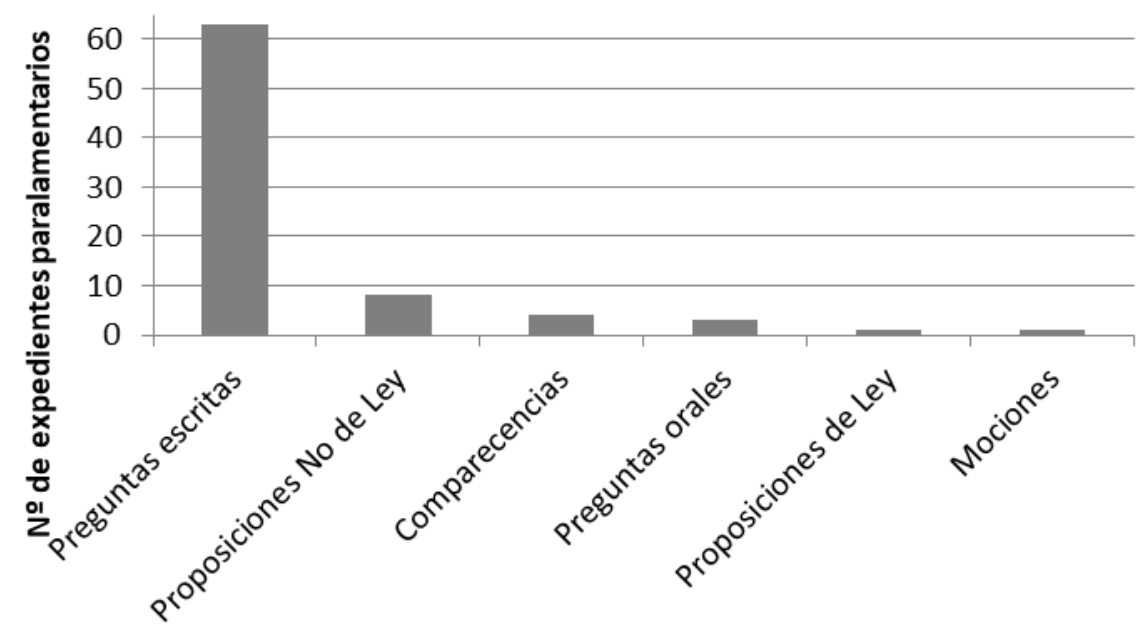

Tipos de iniciativas

Figura 2. Distribución de los expedientes parlamentarios sobre las TAC en la Cortes Generales del Estado español por tipos de iniciativas (1979-2018).

En 2017, el grupo Ciudadanos -de orientación política liberal y que en 2015 obtuvo por primera vez representación en las Cortes Generales- fue la primera formación política con representación nacional en plantear un giro radical en la orientación de las iniciativas parlamentarias sobre las TAC en España. Si hasta ese momento se había estado demandando una regulación que ofreciera seguridad tanto a los posibles pacientes (para evitar riesgos y fraudes) como a los profesionales sanitarios (seguridad jurídica), a partir de 2017 el foco de atención 
se centró exclusivamente en la seguridad de los pacientes. Así, Ciudadanos planteó una PNL "relativa a la mejora de la protección de los pacientes afectados por la pseudociencia" (Congreso de los Diputados, 2017a: 46). A remolque de esta iniciativa, el Grupo Popular -cuyo partido de orientación conservadora estaba en el poder- presentó una nueva propuesta "relativa a las pseudoterapias" (Congreso de los Diputados, 2017b: 24) cuyo texto salió adelante con enmiendas de otros grupos. El último año del periodo analizado, en 2018, desde la oposición el PSOE impulsó una iniciativa para tomar medidas dirigidas a evitar la celebración:

"[...] de congresos acientíficos, así como cualquier tipo de cursos, anuncios, programas en medios de comunicación y redes sociales, que juegan con la esperanza de las personas enfermas, ofreciendo propuestas carentes de cualquier rigor o evidencia" (Congreso de los Diputados, 2018a: 65).

Por último, el grupo Ciudadanos presentó en 2018 una PDL para incluir la "pérdida de oportunidad asistencial por métodos terapéuticos no evaluados ni autorizados" (Congreso de los Diputados, 2018b: 1) como un nuevo delito tipificado en el Código Penal. Esta propuesta está actualmente pendiente de debate y votación.

\subsection{Utilización de significantes}

Dos fueron las fórmulas para referirse a las TAC en el debate político durante el periodo analizado, bien utilizando significantes específicos (SE) referidos TAC concretas, o bien mediante significantes generales (SG) que evidencian una estrategia de generalización.

En cómputos generales, la homeopatía y la acupuntura fueron los SE más recurrentes al mencionarse en 15 y 14 expedientes parlamentarios, respectivamente, y ambas terapias se solaparon en 11 de ellos. Otras TAC presentes en el discurso, pero en menor medida, fueron la naturopatía (6), la medicina tradicional china (4), la quiropraxia (2) y la ayurveda (2). La osteopatía, la hidroterapia y las terapias termales solo se mencionaron una vez en cada caso.

Por lo que respecta a los SG, destacó el uso de los adjetivos calificativos 'natural', 'alternativa', 'no convencional', 'complementaria', 'tradicional' e 'integradora/holística', que se combinaron con sustantivos como 'terapia', 'práctica', 'tratamiento' y 'medicina'. La expresión 'terapias naturales', en singular - plural, fue la más usada por los políticos españoles, estando presente en 31 expedientes, seguida muy de cerca de la expresión 'terapias alternativas' (29) y 'terapias no convencionales' (20). Mucho menos recurrentes fueron otros términos como 'terapias complementarias' (6), 'terapias tradicionales' (5) o 'terapias integradoras o integrativas' (5). En algunos expedientes los políticos combinaron en una misma expresión varios adjetivos, como por ejemplo 'alternativas y/o complementarias', 'alternativas, complementarias o no convencionales', 'naturales 
o alternativas', 'naturales y no convencionales', 'tradicionales o complementarias'. Si bien estos SG podrían calificarse como neutros o positivos, en los dos últimos años al debate sobre las TAC se incorporaron dos nuevos SG -'pseudoterapias' y 'pseudociencias'- que implican connotaciones semánticas negativas y que, por tanto, evidencian un giro radical en la orientación del debate político.

Desde una perspectiva diacrónica, la frecuencia de utilización de SE y SG divide el marco temporal analizado en dos grandes fases: 1) 1984-2006; y, 2) 2007-2018 (ver Figura 1). En la primera fase los políticos españoles articularon el debate en torno a una serie de TAC concretas para las que pedían su regulación, especialmente en el caso de la homeopatía y la acupuntura, y en menor medida, la medicina tradicional china o la quiropraxia. En cambio, en la segunda fase las alusiones a TAC específicas o bien se combinaron con un mayor número de referencias a SG, o bien desaparecieron del debate. Así, la utilización de SE se mantuvo estable en el periodo analizado, salvo en 2017 y 2018, en que tales especificidades se eliminaron. Por el contrario, el uso de SG como estrategia de generalización se registró en los periodos de mayor atención política, concretamente entre 2007 y 2010 y en 2017 y 2018.

Sin embargo, el debate en estos dos periodos presentó dos diferencias clave. En primer lugar, de 2007 a 2010 la utilización de SG estuvo siempre acompañada de SE en la misma línea que en los años anteriores; por el contrario, en los dos últimos años, los SG articularon en solitario el discurso. Segundo, mientras que de 2007 a 2010 solo se utilizaron SG que llevaban años y décadas visitando y revisitando el discurso -como 'terapias naturales', 'alternativas', 'complementarias', etc.-, en 2017 y 2018 aparecieron dos nuevos SG para referirse a las TAC -'pseudociencias' y 'pseudoterapias'-. En consecuencia, la orientación del debate en cada uno de estos dos periodos difirió notablemente. Así, mientras que entre 2007 y 2010 se perseguía establecer un marco regulatorio que redundara en beneficio de los usuarios y de los profesionales que ejercían las TAC, en consonancia con un modelo pluralista, en 2017 y 2018 la desaparición de alusiones a SE y la inclusión de nuevos SG con connotaciones negativas reformularon los límites y objetivos que debía perseguir el diseño de políticas públicas hacia una postura oposicionista.

Dependiendo de la intencionalidad del discurso político en cada momento pluralista u oposicionista-, también se utilizaron otros SG adicionales. Desde la simpatía y tolerancia ante las TAC, los políticos españoles se refirieron a ellas como 'especialidades médicas' (potenciales), 'disciplinas no anecdóticas', 'disciplinas sanitarias', 'técnicas/prácticas terapéuticas', 'terapias individualizadas', 'prácticas médicas y paramédicas' y 'actividades relacionadas con la salud'. Cuando se buscaba la neutralidad, el SG omitía cualquier adjetivo calificativo cuyo significado orientara la postura política, en un sentido u en otro, y simplemente se hablaba de 'actividades/prácticas/campos/técnicas' o 'sector'. Dado que los sustantivos 'pseudoterapias' y 'pseudociencias' no se incorporaron al discurso 
político hasta los últimos años analizados, anteriormente los posicionamientos contrarios a las TAC se introdujeron en el debate con expresiones como 'prácticas fraudulentas' o simplemente 'charlatanería'.

Otra constante del debate político sobre este tema fue que se construyó de forma binaria. Tal dicotomía evidencia el establecimiento de una frontera mediante la yuxtaposición entre los diferentes SE y SG para referirse a las TAC, detallados en los párrafos anteriores, y otra serie de significantes para aludir a su opuesto, es decir, a la medicina de la corriente principal o más generalizada en los países occidentales. En estos casos el sustantivo 'medicina' apareció en el discurso acompañado de adjetivos como 'científica', 'alopática', 'clásica', 'oficial', 'ortodoxa', 'convencional', 'occidental', 'biomédica', 'moderna', 'total' e incluso 'tradicional'; como se ha visto, este último adjetivo también se utilizó referido a las TAC.

\subsection{Elementos narrativos}

En combinación con los significantes identificados en el epígrafe anterior, el debate político de las TAC en España se articuló en torno a una serie de elementos narrativos que, a menudo, aparecieron entrelazados. Entre ellos destacan: 1) la necesidad de regular y el peso de la evidencia científica; 2) la complejidad que entraña la regulación; y, 3) la dualidad del usuario de las TAC como beneficiario o como víctima.

\subsubsection{La necesidad de regular y el peso de la evidencia científica}

Las primeras inquietudes registradas a este respecto datan de 1984, cuando el Grupo Popular, entonces principal partido de la oposición, preguntó al Ejecutivo del Partido Socialista cuándo tenía previsto regular la acupuntura, "como técnica terapéutica eficaz" con la que "se puede ahorrar en el enorme capítulo de gastos farmacéuticos" (Senado de España, 1984: 4609). En 1989 el Grupo Popular reiteró su petición añadiendo la homeopatía. Ante este interés, el Gobierno socialista, reaccionó presentando ese mismo año una PNL para debatir la cuestión en lo referido a la enseñanza y el ejercicio, tanto de la acupuntura como de la homeopatía. La PNL fue aprobada a pesar de la abstención del Grupo Popular que, sorprendentemente y contradiciendo su postura anterior, mantuvo que no había necesidad de regular. Así lo expresó su diputado, Botella Crespo: La regulación sobre técnicas terapéuticas está hecha, y está regulada desde que existe un código deontológico dentro de un organismo que se llama Consejo General de Colegios de Médicos de España. [...] Lo que quiero decirle es que nosotros [Partido Popular] no hemos entendido en absoluto la necesidad de regular algo ya regulado 4 .

\footnotetext{
${ }^{4}$ Diario de Sesiones del Congreso de los Diputados, Comisión de Política Social y de Empleo, de 28 de junio de 1989, núm. 498, p. 16178. Expediente: 161/000227.
} 
En el debate de esta PNL de 1989 el peso de la evidencia científica ya generó dudas entre los legisladores. Así, el Grupo CDS -un partido de centroderecha, que años después desapareció del mapa político- mostró sus reticencias porque consideraba estas 'técnicas' como lo opuesto a la "medicina correcta, rigurosa, científica" ${ }^{5}$. En el mismo sentido, el Grupo Mixto describió la acupuntura como "la expresión local de una técnica que no tiene ninguna base científica" en comparación con la "medicina científica, que da unos razonamientos y ofrece unos resultados" (Diario de Sesiones del Congreso de los Diputados, 1989: $16180)^{6}$.

Como a pesar de la aprobación de esta PNL la regulación de esta materia siguió siendo una asignatura pendiente, en 1994 el Grupo Catalán CiU volvió a preguntar al Ejecutivo del Partido Socialista por la fecha prevista para regular las condiciones para la enseñanza y el ejercicio de la acupuntura, la medicina tradicional china y la homeopatía. La contestación del Gobierno dio a entender que, en ese momento, tal cuestión no era un tema prioritario para los ministerios implicados -Sanidad y Consumo, y Educación y Ciencia-, al tiempo que advertía que:

"La utilización sistemática del término 'Medicina', relacionándolo con otras prácticas que no sea el título universitario de la misma, puede crear confusión a los usuarios del Sistema Sanitario" (Congreso de los Diputados, 1994: 151).

Sin embargo, España, como país miembro de la Unión Europea, se vio obligado ese mismo año a trasponer a la normativa nacional la legislación europea sobre los medicamentos homeopáticos mediante el Real Decreto 2208/1994, de 16 de noviembre. Esto mismo ocurrió en 2018, cuando el Gobierno español también tuvo que trasponer la nueva Directiva 2001/83/CE, con disposiciones específicas para los medicamentos homeopáticos, mediante la Orden SSI/425/2018, de 27 de abril. En cualquier caso, en España los productos homeopáticos nunca han estado cubiertos por las prestaciones del Sistema Público de Salud.

En 2002, en la segunda legislatura en que el Partido Popular estuvo al frente del Gobierno de España, un diputado de este mismo grupo se volvió a interesar por las TAC exponiendo que era "necesario una regulación de ámbito nacional que dé seguridad al ciudadano sobre la profesionalidad y la cobertura legal" (Congreso de los Diputados, 2002a: 107) de estas terapias. En su respuesta, el Ejecutivo dio cuenta de dos actuaciones que estaba llevando a cabo como pasos previos a la regulación: 1) una encuesta para conocer cómo estaban reguladas las TAC en los países de la Unión Europea; y, 2) los primeros contactos con el

\footnotetext{
${ }^{5}$ Diario de Sesiones del Congreso de los Diputados, Comisión de Política Social y de Empleo, de 28 de junio de 1989, núm. 498, p. 16179. Expediente: 161/000227.

${ }^{6}$ Diario de Sesiones del Congreso de los Diputados, Comisión de Política Social y de Empleo, de 28 de junio de 1989, núm. 498, p. 16180. Expediente: 161/000227.
} 
Ministerio de Educación para la regulación educativa de algunas de ellas. Si estas iniciativas se pusieron en marcha cabría interpretar que en ese momento sí existía cierta predisposición a regular con una orientación pluralista, tal y como se desprende del primer párrafo de la respuesta del Gobierno, en la que expresa que:

[...] es consciente, no solamente, de la existencia, sino también del auge de estas 'actividades relacionadas con la salud', y de la aceptación social que se produce solidariamente y de forma proporcional, al crecimiento de las mismas (Congreso de los Diputados, 2002b: 60).

De hecho, al año siguiente este mismo Ejecutivo del Partido Popular aprobó el Real Decreto 1277/2003, de 10 de octubre, sobre autorización de centros, servicios y establecimientos sanitarios, cuyo anexo II incluía el apartado U.101 relativo a las Unidades de terapias no convencionales. Según el Registro General del Ministerio de Sanidad ${ }^{7}$ a fecha julio de 2018, estas unidades especiales están funcionando actualmente en establecimientos sanitarios privados ( 1.178 centros) y, en menor medida, públicos (41 centros). En cualquier caso, la norma que propició la creación de estas unidades dejó un vacío legal en parcelas clave como la titulación específica, más allá de la licenciatura en medicina, y las condiciones de ejercicio requeridas, como los sistemas de acreditación de los profesionales y de los centros sanitarios.

A partir de 2007 la urgencia por regular las TAC se hizo aún más evidente cuando el Gobierno de la Generalidad de Cataluña aprobó el Decreto 31/2007, de 30 de enero, por el que se regulaban las condiciones para el ejercicio de determinadas 'terapias naturales' en su territorio. La norma autonómica mencionaba 13 terapias específicas para las que se establecían los requisitos y los sistemas de acreditación de los profesionales y establecimientos sanitarios. Sin embargo, esta norma fue recurrida por el Gobierno del Partido Socialista y por distintos colegios profesionales, siendo finalmente derogada dos años más tarde por el Tribunal Superior de Justicia de Cataluña por invadir competencias del Estado. Fue el primer intento de regular las TAC de manera global en España; su aprobación en el parlamento regional y su posterior derogación en el ámbito judicial estimuló y, al mismo tiempo, condicionó el debate político sobre la materia en los años sucesivos.

A finales de 2007 se aprobó en el Congreso la PNL presentada por el Grupo IUICV en la que se instaba al Gobierno a la creación de un grupo de trabajo que recabara la información necesaria para acometer una futura regulación que acabara con el vacío legal existente. Con objeto de evitar el fracaso ocurrido con

7 La oferta asistencial se puede consultar http://regcess.mscbs.es/regcessWeb/inicioBuscarCentrosAction.do 
el Decreto catalán, el texto de esta PNL recogía expresamente que el grupo de trabajo tenía que estar formado por expertos del Gobierno estatal y de las Comunidades Autónomas con la participación de los agentes del sector implicados, en alusión a los distintos colectivos de profesionales sanitarios y al sector de las TAC (Congreso de los Diputados 2007).

Al tiempo que el grupo de trabajo formado por expertos avanzaba en sus tareas, el Partido Popular remitió al Gobierno Socialista numerosas preguntas parlamentarias en las que dejaba entrever que no veía necesidad de regular las TAC. Entre otras cuestiones, se pedía al Ejecutivo que explicara qué entendía por 'terapias alternativas' o qué finalidad, objetivos y causas justificaban su regulación. En contraste, desde su postura a favor de la regulación de enfoque pluralista, el Grupo de izquierdas IU-ICV presionaba al Gobierno preguntando sobre el avance de las tareas encomendadas al grupo de expertos o sobre cuestiones técnicas, como la incorporación de algunas TAC a las enseñanzas de formación profesional del Catálogo de Cualificaciones Profesionales.

Finalmente, bajo el título Análisis de la Situación de las Terapias Naturales (Ministerio de Sanidad, 2011), en diciembre de 2011 se presentó el informe elaborado por el grupo de expertos, cumpliendo así lo aprobado en la citada PNL de 2007. Pero al contrario de lo que cabría esperar, su publicación no se plasmó en legislación alguna y la actividad parlamentaria sobre la cuestión se redujo a cuatro iniciativas en el periodo 2011-2015, frente a las 46 registradas en la legislatura anterior.

En 2017 se produjo un giro radical en la necesidad de regular las TAC como elemento narrativo. Si hasta entonces los partidos que reclamaban la regulación lo hacían motivados por la necesidad de establecer un marco jurídico que reconociera determinadas TAC y estableciera los requisitos de formación, titulación y ejercicio, ahora se instaba al Gobierno del Partido Popular a modificar el marco legal para poner coto a las mismas basándose en criterios de evidencia científica. Así, Ciudadanos presentó una PNL "relativa a la mejora de la protección de los pacientes afectados por la pseudociencia", en la que pedía al Ejecutivo que modificara el marco legal para que los profesionales sanitarios tuvieran la obligación de denunciar:

"Las prácticas llevadas a cabo por profesionales, titulados o no titulados, que alejadas de la evidencia científica pudieran causar un perjuicio real en la salud directa de sus pacientes" (Congreso de los Diputados, 2017a: 46).

Es decir, si desde 2003 el requisito legal para ejercer las TAC era contar con una titulación en medicina, lo que se pretendía con esta PNL era limitar esa realidad añadiendo un nuevo requisito: que las TAC que se practicaran fueran seguras y tuvieran un efecto terapéutico demostrado según la evidencia científica disponible. 
Este mismo partido preguntó a la ministra de Sanidad en el Pleno del Congreso por "las medidas previstas por el Gobierno para proteger la salud de los pacientes de los daños producidos por las terapias peligrosas sin evidencias" ${ }^{8}$. También en 2017 pero unos meses después, el Grupo Popular en el Gobierno tomó la iniciativa y presentó su propia PNL "relativa a las pseudoterapias", la única presentada sobre esta materia por este partido conservador. Mientras que la PNL de Ciudadanos no contó con los apoyos necesarios para ser aprobada, el Partido Popular negoció con los otros grupos parlamentarios un texto alternativo que finalmente salió adelante pero sin incluir la obligatoriedad del personal sanitario de denunciar, como demandaba Ciudadanos en su propuesta. En cambio, la PNL aprobada sí hacía hincapié en tres puntos: 1) la promoción, educación y divulgación sobre salud y 'pseudoterapias'; 2) la mejora de la vigilancia y el control sobre centros y personas no acreditadas para evitar la publicidad de 'falsas actividades curativas' y de intrusismo profesional; $y, 3$ ) actualizar el informe sobre 'terapias naturales' de 2011 (Congreso de los Diputados, 2017c: 38-39).

En junio de 2018, y tras ganar una moción de censura, un nuevo Ejecutivo del Partido Socialista, presidido por Pedro Sánchez, sustituyó en el Gobierno al Partido Popular que gobernaba en minoría. La llegada del nuevo Ejecutivo socialista supuso un revulsivo que activó el elemento narrativo de la necesidad casi urgencia- de regular las TAC. Así, solo un mes más tarde de la formación del nuevo Gobierno, su ministro de Ciencia, Innovación y Universidades compareció en el Congreso para informar de las líneas generales de las políticas de su área y, en su intervención, se refirió explícitamente a las 'pseudociencias' en el contexto del anuncio de un plan de educación y cultura científica: "[...] para que los ciudadanos sean capaces de pensar científicamente, de diferenciar entre evidencias y manipulación, de detectar las noticias falsas y los bulos. (...) El plan apoyará acciones de divulgación del pensamiento crítico y estudios para conocer las razones por las que los ciudadanos confían en pseudociencias y proponer estrategias de comunicación efectivas para transmitir las evidencias que las desmontan" .

Solo unas semanas después, el Grupo Ciudadanos presentó en el Congreso una proposición de ley (PDL) para que el Código Penal recogiera como delito contra la salud pública la pérdida de oportunidad terapéutica ocasionada por la difusión pública de información falsa o no contrastada sobre métodos terapéuticos no evaluados ni autorizados por la Agencia Española de Medicamentos y Productos Sanitarios. La pérdida de oportunidad terapéutica se definió en la PDL como:

\footnotetext{
${ }^{8}$ Diario de Sesiones del Congreso de los Diputados, Pleno y Diputación Permanente, 10 de mayo de 2017, núm. 51, p. 28. Expediente: 180/000234.

${ }_{9}^{9}$ Diario de Sesiones del Congreso de los Diputados, Comisión de Ciencia, Innovación y Universidades, 12 de julio de 2018, núm. 574, p. 9. Expediente: 214/000089.
} 
"El abandono de tratamientos con eficacia clínica probada [...] por tratamientos naturales o pseudociencias que no están avaladas e indicadas, científicamente, para tratar sus dolencias" (Congreso de los Diputados, 2018c: 3).

\subsubsection{La complejidad que entraña la regulación}

Las dificultades y dudas a las que se enfrentaron los legisladores se evidencian, en primer lugar, por las reiteradas solicitudes de asesoramiento que, desde la sede parlamentaria, se realizaron a expertos en la cuestión. Para atender estas demandas de asesoramiento técnico se organizaron tres sesiones informativas en 1997 -dos de ellas, sobre homeopatía- y se solicitó la colaboración de dos grupos de expertos para elaborar sendos informes monográficos sobre las TAC en su conjunto; el primero de ellos en 2000 (Martín et al., 2000) y el ya mencionado de 2011 (Ministerio de Sanidad, 2011). Además, como se ha explicado anteriormente, la PNL aprobada en 2017 también recogió entre sus puntos la necesidad de actualizar el informe de 2011, lo que presumiblemente se concretará en la presentación de un tercer informe monográfico.

La complejidad a la hora de regular esta materia quedó plasmada en múltiples ocasiones. Así ocurrió en el debate de la PNL presentada por el Partido Socialista en 1989 para regular la homeopatía y la acupuntura, cuando el representante del Grupo Mixto afirmó que se enfrentaban a un "tema que es espinoso" ${ }^{10}$. Y en 1997 cuando, tras la comparecencia de un experto del Ministerio de Sanidad en una comisión especial del Senado para aclarar dudas técnicas sobre la homeopatía, el representante del Grupo Socialista, Gilbert i Bosch, exclamó:

El primer comentario que se me ocurriría hacer es: ¡Vaya lío! Me parece bastante complicado, y me imagino que legislar sobre este tipo de cosas será doblemente complicado. Por tanto, su intervención [la del experto que comparecía] la tomo más como un aprendizaje que como un planteamiento (Diario de Sesiones de las Cortes Generales, 1997: 26)11.

Diez años más tarde, en 2007, el Ejecutivo socialista de Rodríguez Zapatero utilizó el elemento narrativo de la complejidad para contestar a una pregunta escrita sobre sus previsiones para regular las TAC a nivel nacional. El Gobierno argumentó que el primer escollo estaba precisamente en la definición de estas terapias: "La complejidad del término 'medicina tradicional' y el amplio espectro de prácticas que comprende hace difícil definirlo" (Congreso de los Diputados, 2007b: 138). El segundo problema mencionado fue la carencia de una normativa europea global de referencia por falta de consenso entre los Estados miembros de la Unión Europea. Y a ello se sumaba:

\footnotetext{
${ }^{10}$ Diario de Sesiones de las Cortes Generales, Congreso de los Diputados, 28 de junio de 1989, núm. 498, p. 16179. Expediente: 161/000227.

${ }^{11}$ Diario de Sesiones del Senado, Comisión Especial sobre la Ordenación del Servicio Farmacéutico, 4 de marzo de 1997, núm. 99, p. 26. Expediente: 713/000124.
} 
[...] la diversidad de técnicas y procedimientos existentes en el ámbito de las medicinas alternativas, posibles conflictos de intereses entre profesionales sanitarios y no cualificados que desarrollan estas terapias, eventuales conflictos sociolaborales por las consecuencias de la regulación, etc. (Boletín Oficial de las Cortes Generales, 2007: 138).

Los políticos también tuvieron muchos problemas a la hora de decidir qué significantes de las TAC era mejor utilizar para referirse a la materia, muy conscientes de esa idea de que la elección de la palabra define gran parte de su significado y, por tanto, su posicionamiento en el debate y el curso de acción. Prueba de esta dificultad se halla en la exposición de motivos de la PNL impulsada por el grupo parlamentario de izquierdas IU-ICV en 2007. En dicho texto, el grupo proponente desplegó un repertorio completo de significantes:

A día de hoy nos encontramos con una problemática existente desde hace años, la cual cada vez es más necesaria tratar de forma seria y rigurosa; me estoy refiriendo a las llamadas medicinas complementarias y alternativas (MCA), o a las medicinas tradicionales (MT), o a las medicinas no convencionales (MNC), o a cualquier otro término que significando lo mismo según el país y los analistas que la traten tienen la consideración de instrumentos de salud [...] (Congreso de los Diputados, 2007: 23).

Incluso dos años después de la presentación del informe del Ministerio de Sanidad de 2011, el Gobierno del Partido Popular seguía sin ver despejada la dificultad que entrañaba la regulación de esta materia. Así lo puso de manifiesto la ministra de Sanidad cuando respondió a una pregunta oral en el Pleno, remitida por una diputada del Grupo Mixto, que se interesaba por las previsiones del Ejecutivo para regular las TAC. En cualquier caso, como sabe, este es un tema [la regulación de las TAC] que todavía genera cierto nivel de discusión y que necesita ser madurado, y en ese sentido estamos trabajando en esa línea de acuerdo con los informes recibidos ${ }^{12}$.

La complejidad que entraña la regulación de las TAC no es una cuestión resuelta en la actualidad y parte de esa dificultad radica en la necesidad de diferenciar entre numerosas terapias y sus correspondientes indicaciones terapéuticas atendiendo a las evidencias científicas disponibles en cada caso. La utilización de la etiqueta 'pseudociencias' o 'pseudoterapias' como estrategia de generalización, introducida en el debate político por Ciudadanos en 2017, no ha resuelto esta complejidad a la hora de legislar. Así se desprende de una reciente entrevista realizada al ministro de Ciencia, Innovación y Universidades, Pedro Duque, por la publicación especializada Diario Médico. Al ser preguntado por la fecha en que estaba prevista la publicación del listado de pseudoterapias como

12 Diario de Sesiones del Congreso de los Diputados. Pleno y Diputación Permanente, 25 de septiembre de 2013, núm. 141, p. 17. Expediente: 180/000652. 
parte del plan presentado conjuntamente por su Ministerio y el Ministerio de Sanidad, Duque contestó lo siguiente:

Querríamos tener el listado hoy, o mejor, ayer, pero queremos absoluta exactitud, por lo que hay que dar tiempo. (...). Hay cosas muy obvias, pseudoterapias sobre las que no hay que pensar mucho porque está claro que lo son, pero debemos ser escrupulosos con otras áreas y pretendidas terapias sobre las que puede haber dudas (Plaza, 2019: web).

\subsubsection{La dualidad del usuario como beneficiario y víctima (beneficios/riesgos)}

El tercer elemento narrativo identificado en el debate político de las TAC es la dualidad del usuario/paciente como beneficiario pero también como posible víctima o, lo que es lo mismo, entre beneficios y riesgos. La exposición de motivos de la primera PNL sobre esta materia de 1989 ya se justificaba apelando al rol del usuario como beneficiario de estas terapias. Así los proponentes socialistas, a la sazón en el Gobierno, explicaban que los usuarios estaban ya buscando "tratamientos y diagnósticos mucho más naturales" que, lejos de ser algo "anecdótico" era "un fenómeno que tiene que ser contemplado por los sistemas sanitarios" 13 .

En 1997, con motivo de la celebración de una de las sesiones informativas en el Senado, el experto del Ministerio de Sanidad, José Félix Olalla Marañón, se refirió a esta dualidad en su charla sobre la homeopatía: [...] como una medicina alternativa, una medicina, en principio más segura, menos dañina, pero nos lleva al centro de la polémica sobre la eficacia demostrada. [...] Hay buenos profesionales convencidos de la homeopatía [y] falsos homeópatas y gente que se aprovecha de la situación de desamparo de las personas ${ }^{14}$.

Ese mismo año, el elemento narrativo beneficios/riesgos volvió a aparecer en otra sesión informativa del Senado sobre homeopatía. En esta ocasión a cargo de Syra Peña López, presidenta de la Asociación Española de Farmacéuticos Homeópatas. Como era de esperar dada su afiliación profesional, esta experta reivindicó las virtudes que, según su criterio, ofrecía la homeopatía. Así, habló de: Medicina reactiva que estimula la capacidad de reacción del organismo frente a la enfermedad, [...] tratamiento ideal en todas las edades y personas, [...] huyendo de la terapia tradicional [en alusión a la medicina convencional] basada en gestos de destrucción, inhibición o sustitución ${ }^{15}$.

\footnotetext{
${ }^{13}$ Diario de Sesiones del Congreso de los Diputados, Comisión de Política Social y de Empleo, de 30 de junio de 1989, núm. 498, pp. 16177. Expediente: 161/000227.

${ }^{14}$ Diario de Sesiones del Senado, Comisión Especial sobre la Ordenación del Servicio Farmacéutico, 4 de marzo de 1997, núm. 99, p. 24-26. Expediente: 713/000124.

${ }^{15}$ Diario de Sesiones del Senado, Comisión Especial sobre Ordenamiento del Servicio Farmacéutico, de 29 de abril de 1997, núm. 131, pp. 2-8. Expediente: 715/000067.
} 
En la misma sesión también compareció Carmen Peña López, vocal del Consejo General de Colegios Oficiales de Farmacéuticos Homeópatas, que destacó los riesgos que suponen para los usuarios el intrusismo de personas que ejercen de médicos homeópatas sin tener la acreditación académica para ejercer la medicina, y los riesgos de la publicidad de "productos milagro [...] disfrazados de productos homeopáticos"31 debido a las lagunas existentes en la legislación.

Una década más tarde, en 2007, en una respuesta escrita dirigida a un diputado socialista sobre la previsión de la regulación, el Gobierno contestó que uno de los problemas para la regulación era precisamente determinar si existía o no un beneficio real, pues "persistían grandes incertidumbres sobre la utilidad clínica de la mayoría de estas técnicas"16, es decir, sobre sus beneficios terapéuticos. Como referencia, el Gobierno citó las conclusiones del grupo de expertos que elaboró el primer informe sobre las TAC en España (Martín et al., 2000).

También en 2007, el Grupo Catalán CiU, de corte conservador, preguntó en el Pleno del Senado al entonces ministro de Sanidad, Bernat Soria, sobre la inclusión de la homeopatía en el Sistema Nacional de Salud. Cuando el ministro contestó que no tenía previsto tal actuación, en el turno de contrarréplica la senadora Aleixandre i Cerarols le apremió a hacerlo para acabar con la "indefensión [de los ciudadanos] ante algunos charlatanes" y para "normalizar las terapias naturales que, por desgracia, en algunas ocasiones se utilizan muy mal por algunos de nuestros pacientes" ${ }^{\prime 17}$.

En diciembre de 2007, pero en el Congreso, los grupos parlamentarios recurrieron al elemento narrativo de la dualidad de los usuarios de las TAC como beneficiarios y víctimas en el debate de la PNL presentada por IU-ICV para estudiar y regular la materia. Así, mientras que este grupo, partidario de la regulación con una visión pluralista, se refería a ellas como "un instrumento de salud en términos de prevención, de tratamientos, y de curación de enfermedades", como algo diferente de la "medicina oficial", con "personalidad propia y probada eficacia", el Grupo Popular se quejaba del "montón de indefiniciones sobre la cuestión", aunque finalmente apoyaron la PNL porque era un problema serio que "afectaba a muchos ciudadanos". El Grupo Socialista también puso el acento en el rol de víctimas de los usuarios, pues no "todos los tratamientos llamados hoy terapias naturales son inocuos en todos los casos" ${ }^{18}$.

\footnotetext{
${ }^{16}$ Boletín Oficial de las Cortes Generales, Congreso de los Diputados, 12 de julio de 2007, núm. 586, p. 138. Expediente: 184/119158.

17 Diario de Sesiones del Senado, Sesión del Pleno, 24 de octubre de 2007, núm. 135, p. 8465. Expediente: 680/001441.

18 Diario de Sesiones del Congreso de los Diputados, 11 de diciembre de 2007, núm. 964, pp. 10-16. Expediente: 161/002428.
} 
Esta dinámica argumentativa revisitó el discurso de las TAC en 2009, con motivo del debate de la PNL presentada por el Grupo Socialista para regular que el ejercicio de la homeopatía fuera ámbito exclusivo de los titulados en medicina. En la exposición de motivos de la propuesta se decía: Existe una demanda por parte de la sociedad española que reclama la medicina homeopática realizada por médicos licenciados en medicina [...] y no bajo el oscurantismo y la seudomedicina con la que se suele practicar en otros establecimientos por personas que no son médicos licenciados ${ }^{19}$.

El Grupo Popular, en cambio, intervino en el debate para recalcar que estaba de acuerdo con la iniciativa precisamente para evitar riesgos a los pacientes, pues no solo se trataba "de un posible fraude al consumidor, sino de un verdadero riesgo para el usuario" cuando la homeopatía se utilizaba "para patologías calificadas de graves, y a veces hasta muy graves" ${ }^{\prime 35}$.

En 2017, la tramitación de la PNL presentada por el Grupo Ciudadanos, con objeto de proteger a los pacientes de las pseudoterapias sin evidencia científica, se motivó precisamente aludiendo al problema de los "afectados por la pseudociencia", de "las prácticas [...] que alejadas de la evidencia científica pudieran causar un perjuicio real a la salud de sus pacientes" (Congreso de los Diputados, 2017: 40).

En el debate de esta PNL la noción general y abstracta de víctima se concretó al ser personificada con nombre y apellidos cuando el Grupo Ciudadanos citó como "caso bastante paradigmático" 20 el de Mario Rodríguez. Este joven valenciano falleció de leucemia en 2014 tras abandonar un tratamiento de la medicina convencional por un tratamiento alternativo. Basándose en esta casuística, y aludiendo a otros casos parecidos reportados en la cobertura informativa de los medios de comunicación, Ciudadanos argumentó que era necesario establecer protocolos de denuncias parecidos a los existentes para los casos de violencia de género o abusos a menores. Por tanto, la defensa de esta iniciativa, que encaja en el modelo oposicionista, se argumentó aludiendo a las víctimas del pasado, como Mario, y sobre todo para evitar futuras víctimas. Se trata de una retórica poderosa, por su personificación y dramatismo, que deja poco margen al disenso y la matización.

\section{Conclusiones}

Este estudio analiza el discurso de las TAC en el debate político español durante el periodo 1979-2018 desde el marco general de los Estudios Sociales de la

\footnotetext{
19 Diario de Sesiones del Congreso de los Diputados, Comisión de Sanidad y Consumo, 29 de septiembre de 2009, núm. 351, pp. 13-14. Expediente: 161/001141.

20 Diario de Sesiones del Congreso de los Diputados, Comisión de Sanidad y Servicios Sociales, 21 de septiembre de 2017, núm. 322, p. 14. Expediente: 161/001976.
} 
Ciencia y aplicando técnicas de análisis de contenido y de análisis del discurso, con objeto de medir la atención política dedicada al tema, el léxico y la semántica utilizadas, así como los elementos narrativos que jalonaron casi cuatro décadas de actividad parlamentaria. Los hallazgos de esta investigación conducen a las conclusiones que se detallan a continuación.

En primer lugar, la actividad política sobre esta materia se centró más en la acción de control al Gobierno mediante preguntas escritas y orales que en la presentación de propuestas concretas. Al contrario de lo ocurrido, por ejemplo, en el Parlamento sueco donde se presentaron 99 mociones en el periodo comprendido entre 1980 y 2015 (Danell, 2018). Los dos grandes partidos políticos que históricamente se han turnado en el Gobierno de España desde 1982, el Partido Popular, de corte conservador y el Partido Socialista, socialdemócrata, no han mantenido posturas coherentes a lo largo del periodo estudiado.

Por otra parte, el peso de la evidencia científica en el debate se manifestó por la constante solicitud de asesoramiento experto, bien mediante sesiones informativas o bien mediante la solicitud de informes monográficos a petición del Congreso. Paradójicamente, todos estos esfuerzos de los legisladores por recabar información antes de adoptar una postura no contribuyeron a crear las condiciones de consenso necesarias para la formulación y aprobación de políticas. La complejidad de la materia, que en muchos momentos del debate pareció superar a los representantes políticos, y los intereses contrapuestos de los agentes sociales implicados, junto con los cambios de postura de los principales partidos, pueden haber contribuido a socavar la transcendencia de las aportaciones realizadas por los grupos de expertos.

En cuanto a los diferentes modelos éticos que articulan la relación entre las TAC y la medicina convencional descritos por Kaptchuk y Miller (2005), el debate político en España mantuvo, en general, una orientación pluralista hasta 2017, cuando prácticamente desaparecieron del debate las alusiones a modalidades específicas de TAC. Si hasta esa fecha los significantes generales más utilizados en el discurso para definir estas terapias -como 'naturales', 'alternativas' o 'no convencionales'tenían una carga semántica positiva o neutra que se ajustaba a las características del modelo pluralista, a partir de entonces se introdujeron nuevos significantes 'pseudoterapias' y 'pseudociencias'- con connotaciones negativas más propias del modelo oposicionista. Precisamente estos dos significantes se encuentran entre los cuatro más utilizados por los diarios digitales españoles para referirse a las TAC en el periodo 2015-2017 (Moreno, 2019). Si se atiende al orden cronológico en que ambos términos aparecen en el discurso mediático y político, estos resultados señalarían que el discurso mediático sobre las TAC permeó el discurso político en los dos niveles identificados por Hilgartner y Bosk (1988), es decir, introduciendo el tema en la agenda política y estableciendo una nueva forma de presentar el problema mediante la utilización de estos nuevos significantes. 
Asimismo, la homeopatía y la acupuntura fueron las modalidades específicas que más acapararon la atención de los políticos españoles a la hora de proponer una regulación sobre esta materia, aunque a partir de 2007 lo que se persiguió fue un abordaje global, ya fuera con una orientación pluralista, como ocurrió hasta 2017, o con una orientación oposicionista. También es en 2017 cuando la dualidad narrativa que aludía a los usuarios de las TAC tanto en calidad de beneficiarios como de víctimas desaparece, y las iniciativas propuestas a partir de ese momento tienen como objetivo final la protección del paciente. Así se transitó de la retórica de los riesgos y los beneficios, a la retórica de la seguridad del paciente asociada exclusivamente a la medicina basada en la evidencia.

Por otra parte, las mayorías parlamentarias respondieron de distinta manera a acontecimientos externos en función del contexto. Así, por ejemplo, en el caso del Decreto catalán de 2007, que posteriormente fue anulado por la justicia, reaccionaron logrando un consenso para encargar a los expertos que estudiasen la cuestión y realizasen una propuesta de regulación para todo el territorio nacional. En cambio, la publicación de los trabajos del grupo de expertos en 2011, no se concretó en medidas concretas y el debate permaneció prácticamente dormido hasta que la muerte de Mario Rodriguez en 2014 reintrodujo la cuestión en la agenda mediática. En esta ocasión, el consenso no se construyó retomando las aportaciones del informe de 2011, sino renombrando las TAC como 'pseudociencias' o 'pseudoterapias', sin realizar distinciones ni matizaciones, y demandando una regulación estricta para su control y erradicación, incluso en los casos en que se practiquen por titulados en medicina, que es el límite que establece la legislación vigente. La literatura sobre la cuestión describe cómo en Estados Unidos en los años sesenta y setenta del siglo pasado los editoriales de las revistas médicas de más prestigio también utilizaron como elemento dramático la noción de víctima de las TAC para presionar a los políticos a la hora de demandar una legislación oposicionista (Winnick, 2005).

Al contrario que en décadas anteriores, en la actualidad no parece existir espacio discursivo para posicionamientos políticos matizados, ya sea por tipos de terapias o por tipos de patologías, o que otorguen más peso a cuestiones como los beneficios en términos de bienestar o inocuidad de algunas de ellas, cuestión ya destacada entre las conclusiones de los grupos de expertos (Ministerio de Sanidad, 2011). También se han eliminado del debate político las referencias a la relación entre el uso de las TAC y los cambios en el sistema de valores y creencias, es decir, la dimensión cultural de la cuestión.

Siguiendo el planteamiento de Laclau (2005) en su análisis del fenómeno del populismo político, si entre los populismos de izquierda y de derecha existe una tierra de nadie que muchas veces es cruzada, en el actual debate político de las TAC en España parece existir esa misma polarización entre lo que representa la medicina convencional y lo que representan las TAC, con una tierra de nadie 
cuyas trincheras y alambradas solo pueden salvarse con el respaldo de la evidencia científica.

\section{Agradecimientos}

El presente trabajo ha sido desarrollado en el marco de dos proyectos de investigación: "Estudio y clasificación de las terapias naturales, complementarias y alternativas a través de los medios de comunicación y de las redes sociales. Ideas y valores de transferencia al imaginario social" (CSO2014-57778-R), financiado por el Ministerio de Economía y Competitividad y por Fondos FEDER de la Unión Europea, y el proyecto "Praxeología de la cultura científica: evaluación y medición" (FFI2017-82217-C2-2-P), financiado por el Ministerio de Economía y Competitividad. Se agradece, asimismo, el trabajo de los revisores cuyas aportaciones han permitido mejorar el manuscrito. 


\section{Referencias bibliográficas}

AGENCIA ESTATAL BOLETÍN OFICIAL DEL ESTADO (2003): Boletín Oficial del Estado: jueves 23 de octubre de 2003, Núm. 254. Pp. 37902. Disponible en: https://www.boe.es/boe/dias/2003/10/23/pdfs/A37893-37902.pdf

ALMEIDA, J., SIEGEL, P., y BARROS, N. (2018): Towards the glocalisation of complementary and alternative medicine: Homeopathy, acupuncture and traditional Chinese medicine practice and regulation y Brazil and Portugal, en BROSNAN, C., VUOLANTO, P. y DANNELL, J. (Eds.): Complementary and alternative medicine. Health, technology and society, Palgrave Macmillan, pp. 113-137.

BAÑÓN, A. (2013): Salud y discurso. A modo de introducción. Discurso \& Sociedad, 7 (1), pp. 1-25.

BAÑÓN, A., FORNIELES, J., SOLVES, J., y RIUS, I. (2011) (Coords): Desafíos y estrategias comunicativas de las enfermedades raras: la investigación médica como referente. Estudio cuantitativo-discursivo de la prensa escrita impresa y digital española (2009-2010). Valencia, CIBERER.

BAYLEY, P. (2004): Cross-cultural perspectives on parliamentary discourse. Amsterdam/Philadelphia, John Benjamins Publishing Company.

BROOKS, H. (1964): The scientific advisor, en GILPIN, R. y WRIGHT, C. (Eds.): Scientists and national policymaking. New York, Columbia University Press, pp. 73-96.

BROSNAN, C. y KIRBY, E. (2016): Sociological perspectives on the politics of knowledge in health care: introduction to themed issue. Health Sociology Review, 25 (2), pp. 139-141.

BROSNAN, C., VUOLANTO, P. y DANNELL, J. (2018): Introduction: Reconceptualising complementary and alternative medicine as knowledge production and social transformation, en BROSNAN, C., VUOLANTO, P. y DANNELL, J. (Eds.): Complementary and alternative medicine. Health, technology and society, Palgrave Macmillan, pp. 1-29.

CANO-ORÓN, L. (2016): Correlación entre las búsquedas sobre terapias complementarias en Google y su uso por parte la población española. Panacea, XVII (44), pp. 124-132.

CANO-ORÓN, L. y MORENO, C. (2017): Las terapias no convencionales en la campaña de las elecciones generales de 2015. Revista de Comunicación y Salud, v. 7, pp. 29-47. 
CAUFFIELD, J. (2000): The psychosocial aspects of complementary and alternative medicine. Pharmacotherapy, 20 (11), pp. 1289-1294.

CIS (CENTRO DE INVESTIGACIONES SOCIOLÓGICAS) (2018): Barómetro de febrero 2018. Núm. 3205. Disponible en: http://www.cis.es/cis/opencm/ES/1_encuestas/estudios/ver.jsp?estudio=14383 [Consultado 12-12-2018].

CONGRESO DE LOS DIPUTADOS (1994): Boletín Oficial de las Cortes Generales, 31 de octubre de 1994, núm. 155, p. 151. Expedientes: 184/005801 y 184/005804.

(2002a): Boletín Oficial de las Cortes Generales, 27 de marzo de 2002, núm. 331, p. 107. Expediente: 184/026881.

(2002b): Boletín Oficial de las Cortes Generales, 26 de septiembre de 2002, núm. 410, p. 60. Expediente: 184/026881.

(2007a): Boletín Oficial de las Cortes Generales, 20 de diciembre de 2007, núm. 652, pp. 16-17. Expediente: 161/002428.

(2007b): Boletín Oficial de las Cortes Generales, 12 de julio de 2007, núm. 586, p. 138. Expediente: 184/119158.

(2007c): Boletín Oficial de las Cortes Generales, 19 de octubre de 2007, núm. 622, p. 23. Expediente: 161/002428.

(2017a): Boletín Oficial de Las Cortes Generales, 28 de febrero de 2017, núm. 112, p. 46. Expediente: 161/001403.

CONGRESO DE LOS DIPUTADOS (2017b): Boletín Oficial de las Cortes Generales, 20 de junio de 2017, núm. 177, p. 24. Expediente: 161/001976.

(2017c): Boletín Oficial de las Cortes Generales, 18 de octubre de 2017, núm. 231, pp. 38-39. Expediente: 161/001976.

(2017d): Boletín Oficial de las Cortes Generales, 18 de octubre de 2017, núm. 231, p. 40. Expediente: 161/001976.

(2018a): Boletín Oficial de las Cortes Generales, 20 de febrero de 2018, núm. 301, p. 65. Expediente: 161/002904.

(2018b): Boletín Oficial de las Cortes Generales, 7 de septiembre de 2018, núm. 304-1, p. 1. Expediente: 122/000226. 
(2018c): Boletín Oficial de las Cortes Generales, 7 de septiembre de 2018, núm. 304-1, p. 3. Expediente: 122/000266.

DANNELL, J. (2018): Translation of complementary and alternative medicine in Swedish politics, en BROSNAN, C., VUOLANTO, P. y DANNELL, J. (Eds.): Complementary and alternative medicine. Health, technology and society, Palgrave Macmillan, pp. 165-191.

DÍAZ, J. (2006): Cómo denominar a las «otras» medicinas, en FACULTAD DE CIENCIAS DE LA INFORMACIÓN (Coord.): El Cajetín de la Lengua-Espéculo Revista de Estudios Literarios. Facultad de Ciencias de la Información de la Universidad Complutense de Madrid (España). Disponible en: https://webs.ucm.es/info/especulo/cajetin/medalter.html [Consultado 1-02-2019].

DIJK, T. (2008): Semántica del discurso e ideología. Discurso y Sociedad, 2 (1), pp. 201-261.

EISENBERG, D., KESSLER, R., VAN, M., KAPTCHUK, T., WILKEY, S., APPEL, S. y DAVIS, RB. (2001): Perceptions about complementary therapies relative to conventional therapies among adults who use both: Results from a national survey. Annals of Internal Medicine, 135 (5), pp. 344-351.

ENTMAN, R. (2004): Projections of power: framing news, public opinion and US foreign policy. Chicago, University of Chicago Press, U.S.A.

ESCRIBÀ-SALES, E., ROVIRA, S. y ALONSO-MARCO, F. (2015): Pseudoscience in the media: A case study of homeopathy as covered in the Spanish and British press (2009-2014). Panacea, 16 (42), pp. 177-183.

EUROPEAN PARLIAMENT (POLICY DEPARTMENT A: ECONOMIC AND SCIENTIFIC POLICY) (2017): Complementary and alternative therapies for patients today and tomorrow. Disponible en: http://www.europarl.europa.eu/RegData/etudes/STUD/2017/614180/IPOL_STU(2 017)614180_EN.pdf [Consultado 03-07-2018].

GALE, N. (2014): The Sociology of Traditional, Complementary and Alternative Medicine. Sociology Compass, 8 (6), pp. 805-822.

GEIST-MARTIN, P. y BELL, K. (2009): 'Open Your Heart First of All': Perspectives of Holistic Providers in Costa Rica About Communication in the Provision of Health Care. Health Communication, 24 (7), pp. 631-646.

HAN, E., JOHNSON, N., DELAMELENA, T., GLISSMEYER, M. y STEINBOCK, K. (2011): Alternative Therapy Used as Primary Treatment for Breast Cancer Negatively Impacts Outcomes. Annals of Surgical Oncology, 18 (4), pp. 912-916. 
HILGARTNER, S. y BOSK, C. (1988): The rise and fall of social problems: a public arenas model. The American Journal of Sociology, 94 (1), pp. 53-78.

HONDA, K. y JACOBSON, J. (2005): Use of complementary and alternative medicine among United States adults: The influences of personality, coping strategies, and social support. Preventive Medicine, 40 (1), pp. 46-53.

IJAZ, N., BOON, H., WELSH, S. y MEADS, A. (2015): Supportive but 'worried': Perceptions of naturopaths, homeopaths and Chinese medicine practitioners through a regulatory transition in Ontario, Canada. BMC, Complementary and Alternative Medicine, v. 15, p. 312, doi: 10.1186/s12906-015-0846-6

JASANOFF, S. (2004): Ordering knowledge, ordering society, en JASANOFF, S. (Ed.): States of knowledge. The coproduction of science and social order. Londres, Routledge, pp. 13-45.

KAPTCHUK, T. y MILLER, F. (2005): What is the best and most ethical model for the relationship between mainstream and alternative medicine: Opposition, integration, or pluralism? Academic Medicine, 80 (3), pp. 286-290.

KOHATSU, N., ROBINSON, J. y TORNER, J. (2004): Evidence-based public health - An evolving concept. American Journal of Preventive Medicine, 27 (5), pp. 417421.

LACLAU, E. (2005): La razón populista. Buenos Aires, Argentina, Fondo de Cultura Económica de Argentina.

LEEUWEN, T. (2005): Introducing Social Semiotics. Oxon, Routledge.

LEGGETT, S., KOCZWARA, B. y MILLER, M. (2015): The Impact of Complementary and Alternative Medicines on Cancer Symptoms, Treatment Side Effects, Quality of Life, and Survival in Women With Breast Cancer-A Systematic Review. Nutrition and Cancer-an International Journal, 67 (3), pp. 373-391.

MARTíN, J., GÓNGORA, F., YÁÑEZ, D., MIGUÉLEZ, C. y GONZÁLEZ, J. (2000): Medicinas alternativas: descripción general. Informe técnico de la Agencia de Evaluación de Tecnologías Sanitarias (AETS). Madrid, Ministerio de Sanidad y Consumo.

MCCOMAS, K. y SHANAHAN, J. (1999): Telling stories about global climate change - Measuring the impact of narratives on issue cycles. Communication Research, 26 (1), pp. 30-57.

MINISTERIO DE SANIDAD, CONSUMO Y BIENESTAR SOCIAL y MINISTERIO DE CIENCIA, INNOVACIÓN Y UNIVERSIDADES (2018): Plan para la protección de la 
salud frente a las pseudoterapias. Pp. 1-13. Disponible en: http://www.ciencia.gob.es/stfls/MICINN/Ministerio/FICHEROS/20181108_Plan_Pr oteccion_frente_pseudoterapias_VF.pdf

MINISTERIO DE SANIDAD, POLÍTICA SOCIAL E IGUALDAD (2011): Análisis de la situación de las terapias naturales. Disponible en: https://www.mscbs.gob.es/novedades/docs/analisisSituacionTNatu.pdf [Consultado 02-07-2018].

MINISTERIO DE SANIDAD, POLÍTICA SOCIAL E IGUALDAD (2013): Encuesta Nacional de Salud 2011/12. Disponible en: https://www.mscbs.gob.es/estadEstudios/estadisticas/encuestaNacional/encuesta 2011.htm [Consultado 15-05-2018].

MORENO, C. (2016) (Ed.): Campañas institucionales en salud pública. El caso de la vacuna contra el VPH. Madrid, Dextra Editorial, S.L.

MORENO, C. y LOPERA, E. (2016): Comparative study of the frequency of use of natural therapies among the Spanish population and their public image in digital media. 14th International Public Communication of Science and Technology Conference (PCST), Estambul, Turquía, pp. 1-4.

MORENO, S. (2019): Estudio sobre el uso de la evidencia científica como argumento en los relatos periodísticos sobre terapias complementarias en los principales diarios digitales españoles (2015-2017). Dígitos, Revista de Comunicación Digital, v. 5, pp. 122-152. Disponible en: https://revistadigitos.com/documentos/Moreno2019_preprint.pdf [Consultado 2001-2019].

MULKAY, M. (1993): Rethorics of hope and fear in the great embryo debate. Social Studies of Science, 23 (4), pp. 721-742.

NOVOA, A. (2019): La construcción social del conocimiento y la importancia del testimonio: a propósito de la vacuna del VPH. Disponible en: http://www.nogracias.eu/2019/01/06/la-construccion-social-del-conocimiento-laimportancia-del-testimonio-proposito-la-vacuna-del-vph-abel-novoa/\#comments [Consultado 20-01-2019].

OMS (2013): Estrategia de la OMS sobre Medicina Tradicional 2014-2023. Disponible en: http://apps.who.int/medicinedocs/documents/s21201es/s21201es.pdf [Consultado 15-10-2018]. 
PATEL, S., KEMPER, K. y KITZMILLER, J. (2017): Physician perspectives on education, training, and implementation of complementary and alternative medicine. Advances in Medical Education and Practice, v. 8, pp. 499-503.

PÉREZ, M. (2008): Discusiones teóricas y metodológicas sobre el estudio del discurso desde el campo de la comunicación. Comunicación y Sociedad, v. 10, pp. 225-247.

PLAZA, J. (2019): Entrevista a Pedro Duque, ministro de Ciencia, Innovación y Universidades: "Lo que la ciencia no valida no cabe en el sistema sanitario; la homeopatía, con los caramelos", en Diario Médico, 8 de enero, sección Sanidad. Disponible en: https://www.diariomedico.com/investigacion/las-pseudoterapiasafectan-poco-al-sistema-sanitario-pero-debemos-lograr-que-no-afecten-nada.html [Consultado 15-01-2019].

SCHREIBER, L. (2005): The importance of precision in language: Communication research and (so-called) alternative medicine. Health Communication, 17 (2), pp. 173-190.

SENADO DE ESPAÑA (1984): Boletín Oficial de las Cortes Generales, Senado. 5 de diciembre de 1984, núm. 116, p. 4609. Expediente: 684/002277.

VV.AA. (2013). Salud y Discurso (número especial). Discurso \& Sociedad, 7 (1).

WEBB, L., PERRY-PARRISH, C., ELLEN, J. y SIBINGA, E. (2018): Mindfulness instruction for HIV-infected youth: a randomized controlled trial. Aids CarePsychological and Socio-Medical Aspects of Aids/Hiv, 30 (6), pp. 688-695.

WEEKS, L. y STRUDSHOLM, T. (2008): A scoping review of research on complementary and alternative medicine (CAM) and the mass media: Looking back, moving forward. BMC, Complementary and Alternative Medicine, v. 8, pp.19.

WINNICK, T. (2005): From quackery to 'complementary' medicine: The American medical profession confronts alternative therapies. Social Problems, 52 (1), pp. 3861.

YUN, Y., LEE, Myung K., PARK, S., KIM, Y., HEO, D., KIM, H. y PARK, S. (2013): Effect of complementary and alternative medicine on the survival and healthrelated quality of life among terminally ill cancer patients: a prospective cohort study. Annals of Oncology, 24 (2), pp. 489-494. 